\title{
Using Cortical Neuron Markers to Target Cells in the Dorsal Cochlear Nucleus
}

\author{
Thawann Malfatti, ${ }^{1}$ Barbara Ciralli, ${ }^{1}$-Markus M. Hilscher, ${ }^{2}$ Steven J. Edwards, ${ }^{3}$ Klas Kullander, ${ }^{3}$
} Richardson N. Leao, ${ }^{1}$ and Katarina E. Leao ${ }^{1}$

https://doi.org/10.1523/ENEURO.0413-20.2020

${ }^{1}$ Hearing and Neuronal activity Lab, Brain Institute, Federal University of Rio Grande do Norte, Natal, Brazil 59056-450, ${ }^{2}$ Institute for Analysis and Scientific Computing, Vienna University of Technology, Vienna, Austria 1040, and ${ }^{3}$ Unit of Developmental Genetics, Department of Neuroscience, Uppsala University, Uppsala, 75237 Sweden

\section{Visual Abstract}

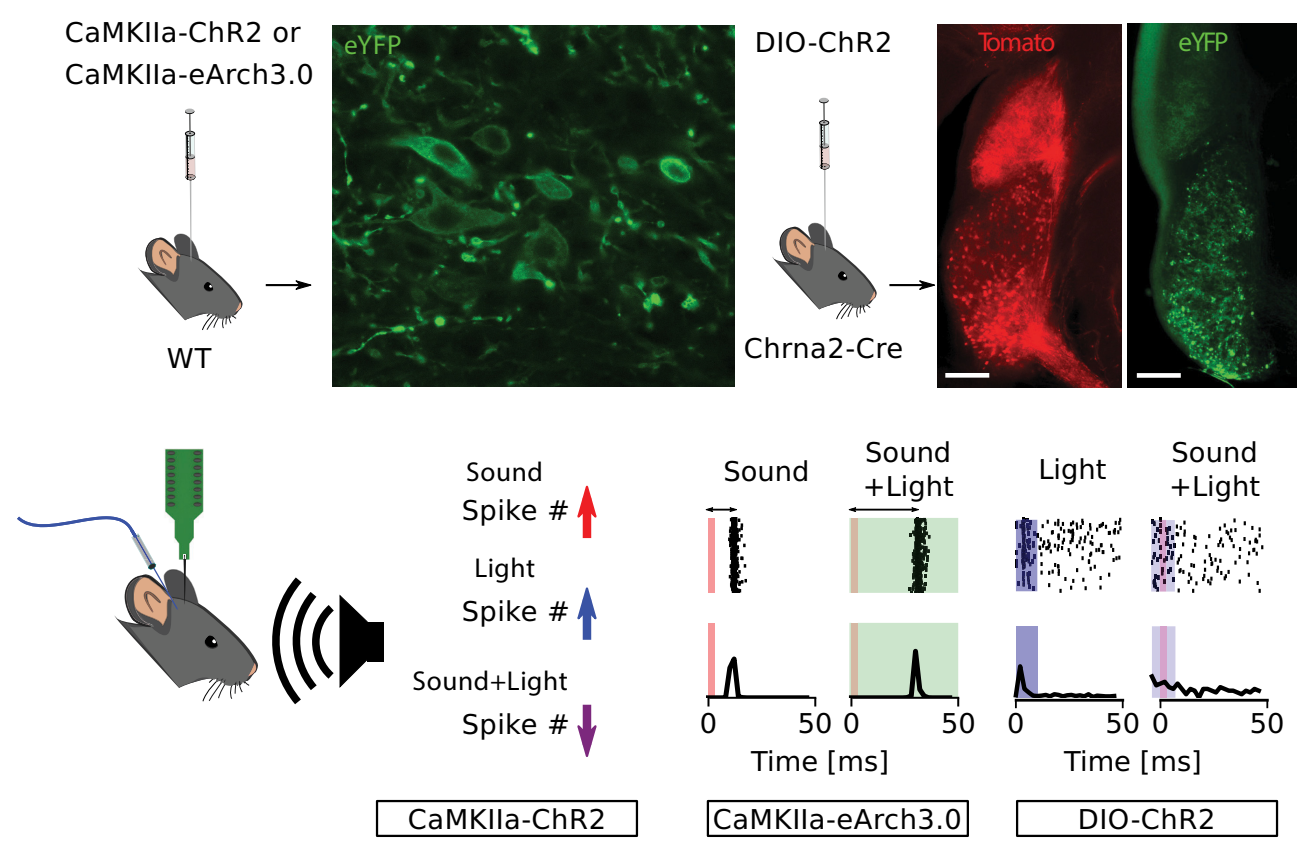

The dorsal cochlear nucleus (DCN) is a region of particular interest for auditory and tinnitus research. However, lack of useful genetic markers for in vivo manipulations hinders elucidation of the DCN contribution

\section{Significance Statement}

Cortical neuron markers could be used to label subcortical regions such as the dorsal cochlear nucleus $(\mathrm{DCN})$ that integrates sound and somatosensory input. Here, we examine whether excitatory (CaMKII $\alpha$ ) or inhibitory [nicotinic acetylcholine receptor $\alpha 2$ subunit (Chrna2)] neuron markers used in neo/paleo-cortex studies label unique DCN populations. We found CaMKII $\alpha$ to be expressed by different DCN neuron populations (affecting sound sensitive and nonsensitive neurons). The Chrna2 promoter was specifically expressed in excitatory cells of the ventral cochlear nucleus (VCN) and could drive indirect activity in the DCN. This study highlights novel ways of regulating DCN neuron activity, which can provide new means for treatment of bothersome tinnitus. 
to tinnitus pathophysiology. This work assesses whether adeno-associated viral vectors (AAV) containing the calcium/calmodulin-dependent protein kinase $2 \alpha$ (CaMKII $\alpha$ ) promoter and a mouse line of nicotinic acetylcholine receptor $\alpha 2$ subunit (Chrna2)-Cre can target specific DCN populations. We found that CaMKIl $\alpha$ cannot be used to target excitatory fusiform DCN neurons as labeled cells showed diverse morphology indicating they belong to different classes of DCN neurons. Light stimulation after driving Channelrhodopsin2 (ChR2) by the CaMKIl $\alpha$ promoter generated spikes in some units but firing rate decreased when light stimulation coincided with sound. Expression and activation of CaMKIl $\alpha$-eArchaerhodopsin3.0 in the DCN produced inhibition in some units but sound-driven spikes were delayed by concomitant light stimulation. We explored the existence of Cre + cells in the DCN of Chrna2-Cre mice by hydrogel embedding technique (CLARITY). There were almost no $\mathrm{Cre}+$ cell bodies in the DCN; however, we identified profuse projections arising from the ventral cochlear nucleus (VCN). Anterograde labeling in the VCN revealed projections to the ipsilateral superior olive and contralateral medial nucleus of the trapezoid body (MNTB; bushy cells), and a second bundle terminating in the DCN, suggesting the latter to be excitatory Chrna2+ T-stellate cells. Exciting Chrna2+ cells increased DCN firing. This work shows that cortical molecular tools may be useful for manipulating the DCN especially for tinnitus studies.

Key words: CaMKIla; Chrna2; dorsal cochlear nucleus; extracellular recording; optogenetics; ventral cochlear nucleus

\section{Introduction}

The dorsal cochlear nucleus (DCN) of the auditory brainstem is the first integrator of auditory and multisensory signals and has been pointed as a key structure in tinnitus physiopathology (Kaltenbach et al., 2005; Tzounopoulos, 2008; Baizer et al., 2012). Cells in the DCN receive direct or indirect [e.g., relayed by the ventral cochlear nucleus (VCN)] sound input onto different cell populations in a layer arrangement. The most cell-populated DCN field is the fusiform cell layer formed by excitatory fusiform cells intercalated with interneurons (Oertel and Young, 2004). An interesting aspect of the DCN is its architectural similarity to the cerebellum (Devor, 2000) that is thought to be responsible for integrative processing (e.g., sound/somatosensory; Oertel and Young, 2004).

Abnormal sensory integration in the DCN is clinically relevant because of the prevalence of temporomandibular tinnitus (Levine, 1999; Grossan and Peterson, 2017). Other forms of mechanical tinnitus are also attributed to aberrant activity in the DCN (Han et al., 2009). Also, a large number of studies have shown altered synaptic and intrinsic cellular properties within the DCN circuit relating

Received September 24, 2020; accepted December 23, 2020; First published February 9, 2021

The authors declare no competing financial interests.

Author contributions: T.M., R.N.L., and K.E.L. designed research; T.M. and B.C. performed research; T.M., M.M.H., S.J.E., and K.K. analyzed data; T.M., B.C., M.M.H., R.N.L., and K.E.L. wrote the paper.

This work was supported by Coordination for the Improvement of Higher Education Personnel (CAPES), the National Council for Scientific and Technological Development (CNPq), and the American Tinnitus Association (ATA).

Acknowledgements: We thank Dr. Helton Maia, Dr. George Nascimento, and $\mathrm{Dr}$. João Bacelo for technical advice

Correspondence should be addressed to Katarina E. Leao at katarina. leao@neuro.ufrn.br

https://doi.org/10.1523/ENEURO.0413-20.2020

Copyright $@ 2021$ Malfatti et al.

This is an open-access article distributed under the terms of the Creative Commons Attribution 4.0 International license, which permits unrestricted use, distribution and reproduction in any medium provided that the original work is properly attributed. to noise-induced tinnitus (for review, see Shore et al., 2016) yet tinnitus treatments to date do not specifically target this region. The VCN can also contribute to noiseinduced tinnitus (Kraus et al., 2011; Coomber et al., 2015). Aberrant activity in any cochlear nucleus subregions can trigger upstream changes as cochlear nucleus neurons relay auditory signals to higher areas of the auditory pathway (Kraus et al., 2011; Coomber et al., 2015). Abnormal activity from auditory cortex and inferior colliculus can also produce downstream alterations in the DCN as its cells receive feedback through descending auditory fibers (Winer and Prieto, 2001; Milinkeviciute et al., 2017). Despite its physiological importance and its well accepted role in tinnitus, the contribution of specific DCN populations to hearing and tinnitus pathophysiology are largely unknown.

Because of its variety of cell types and its cerebellum like structure, DCN circuit studies could benefit from identifying key neuronal markers (Hilscher et al., 2017). The Calcium/ calmodulin-dependent protein kinase $2 \alpha$ (CaMKII $\alpha$ ) promoter is widely used for targeting cortical pyramidal cells. Immunohistochemical data from rats has shown CaMKII $\alpha$ expression in the DCN molecular and fusiform cell layers (Ochiishi et al., 1998). In mice CaMKIl $\alpha$ RNA is widely distributed in the fusiform layer (Lein et al., 2007). Hence, viral vectors to express reporter or optogenetic proteins by the $\mathrm{CaMKIl} \alpha$ promoter may be applied to DCN manipulation. Cortical interneuron markers could also be used to tag DCN cells. However, for two commonly used markers, both parvalbumin and somatostatin expression can be quite promiscuous in the neocortex/hippocampus region (Kawaguchi and Kondo, 2002; Mikulovic et al., 2015) and in the DCN, parvalbumin is distributed across layers without apparent population specificity and somatostatin shows weak expression (Lein et al., 2007). Nonetheless, recently, the nicotinic acetylcholine receptor $\alpha 2$ subunit (Chrna2) has been described as a marker for highly specific interneuron populations (L5 Martinotti cells in the neocortex or CA1 oriens-lacunosum moleculare cells in the ventral hippocampus; Leão et al., 2012; Hilscher et al., 2017). 
Cre + cells in Chrna2-cre mice seem also to belong to single populations in several subcortical nuclei (Mikulovic et al., 2018; Siwani et al., 2018). Whether Chrna2 is expressed in the DCN is not described.

Here, we test whether adeno-associated viral vectors (AAV) with the CaMKII $\alpha$ promoter can be used for manipulating DCN circuits in vivo. AAV encoded optogenetic protein expression and light stimulation paired with brief sound presentation was used to functionally identify cells and assess the effect of optical depolarization/hyperpolarization in input/output functions in CaMKII $\alpha+$ neurons in combination with brief sound stimulation. Lastly, we examined how activation of Chrna2 + cells innervating the DCN modulate DCN unit activity.

\section{Materials and Methods}

\section{Mice}

Male C57BI/6J mice, Chrna2-cre or Chrna2-cre mice crossed with the reporter line Ai14 tdTomato (Chrna2Cre/R26 $\left.{ }^{\text {tom }}\right)$ mice age postnatal day (P)21-P75 $(n=20)$ were used in this study. All animal procedures were approved by the Federal University of Rio Grande do Norte Ethical Committee in Use of Animals (CEUA - protocol number 051/2015) and followed the guidelines for care and usage of laboratory animals of the Federal University of Rio Grande do Norte.

\section{Virus injection of optogenetic constructs}

Approximately fourweeks before experiments using optogenetic stimulation, mice were injected with viral constructs of different opsins coupled to either channelrhodopsin2 (ChR2) or Archaerhodopsin3.0 (eArch3.0). ChR2 is a light activated cation permeable channel (Boyden et al., 2005) for membrane depolarization, while eArch3.0 is a green light activated outward proton $\left(\mathrm{H}^{+}\right)$pump (Chow et al., 2010) for membrane hyperpolarization. ChR2 constructs used were: $\mathrm{rAAV} 5 / \mathrm{CaMKIl} \alpha$-hChR2(H134R)-EYFP (Vector core, at a concentration of $4 \times 10^{12}$ virus molecules $-\mathrm{vm} / \mathrm{ml}$ ) and a cre-dependent (double-floxed inverted open reading frame; DIO) ChR2 construct, AAV2/9. EF1a.DIO.hChR2(H134)-eYFP-WPRE-hGH (Vector core, at $\left.1 \times 10^{13} \mathrm{vm} / \mathrm{ml}\right)$. The eArch3.0 used was rAAV5/ CamK2a-eArch3.0-eYFP (Vector core, at $2.5 \times 10^{12} \mathrm{vm} /$ $\mathrm{ml})$. In detail, mice were anesthetized with ketamine-xylazine at $90 / 6 \mathrm{mg} / \mathrm{kg}$ intraperitoneal. If required, additional ketamine was re-administered (as half the dose of the previous injection, i.e., 45 and $22.5 \mathrm{mg} / \mathrm{kg}$ ) during surgery. The mouse was mounted into a stereotaxic device while resting on a heating block at $37^{\circ} \mathrm{C}$. Eye gel (dexpanthenol) was applied to avoid drying of eyes during surgery. The head was wiped with polyvidone-iodine $(10 \%)$ to avoid infections. The skin was anesthetized with lidocaine hydrochloride 3\% before a straight incision was made. After the incision, hydrogen peroxide $3 \%$ was applied onto the exposed skull to remove the connective tissue and to visualize sutures.

The DCN coordinates were taken from Franklin and Paxinos (2008). Specifically, we used $-6.1 \mathrm{~mm}$ anteroposterior (AP), $-2.3 \mathrm{~mm}$ mediolateral (ML), and -4.3 and
$-3.8 \mathrm{~mm}$ dorsoventral (DV; two steps). For each animal, those coordinates were corrected by multiplying by the normalized bregma-lambda distance (mouse's bregmalambda in mm divided by 4.2 - the average bregma-lambda distance from the mice used in Paxinos and Franklin's atlas), to account for head size differences. Additionally, the vertical distance between the bregma and the point in the skull at the AP and ML coordinate was subtracted from the DV coordinate, so that the DCN can be reached using the brain surface as reference. A small mark was made at the AP and ML coordinates and a small hole was carefully drilled with a dental microdrill (Beavers Dental). Next, prealiquoted virus (20\% for Cre-dependent, 30\% for $\mathrm{CaMKIl} \alpha$-dependent vectors) was rapidly thawed and withdrawn into a microsyringe ( $10 \mu \mathrm{l}$ Nanofil, 34-gauge removable needle), at the speed of $1.5 \mu \mathrm{l} / \mathrm{min}$ using a infusion pump (Chemyx NanoJet). The syringe was lowered into the DCN to the deepest DV coordinate, and $0.75 \mu \mathrm{l}$ of virus was slowly infused at $0.15 \mu \mathrm{l} / \mathrm{min}$ and the needle was kept in place for $5 \mathrm{~min}$ to allow for full diffusion of virus, then retracted to the second, more superficial DV coordinate for a second infusion $(0.75 \mu \mathrm{l})$ of virus and the needle kept in place for $10 \mathrm{~min}$, before carefully removed. Some animals received bilateral injections. Next, the skin was sutured, lidocaine hydrochloride $3 \%$ was applied over the suture and $200 \mu \mathrm{l}$ of saline was injected subdermal in the back for rehydration. The animal was removed from the stereotaxic frame and placed under a red heat lamp and monitored until recovering from anesthesia. Some initial experiments used fluorescent retrobeads (Green fluorescent retrobeads, Lumafluore) to establish the appropriate coordinates of injection. The benefits of initially using retrobeads, compared with viral injections of optogenetic material during optimization of experimental procedures, are (1) the fluorescent liquid can be readily seen withdrawn into the microsyringe with the naked eye (compared with a minute volumes of transparent viral solution that sometimes fails to be withdrawn because of technical issues), and (2) animals can be killed after only a few days (compared with waiting two to four weeks for viral expression) to confirm the appropriate location of fluorescent signal.

\section{Sound calibration and sound stimulation}

As different sound devices can have inherent shifts in unit level, and thereby in the signal generation, the sound card (Sound Devices USBPre2, Thomann GmbH; 192 kHz sampling rate, 24-bit $A D C$ ) was initially calibrated using an oscilloscope. A $10 \mathrm{kHz}$ sine wave of $1 \mathrm{~V}$ amplitude was written to the card, and the sound card output amplification factor was recorded as 1 divided by the amplitude of the output signal. All sound signals were multiplied by the output amplification factor before being written to the card. We connected the sound card output to the sound card input, and a $1 \mathrm{~V} 10 \mathrm{kHz}$ sine wave was played and recorded. The input amplification factor was measured as 1 divided by the amplitude of the recorded signal, and signals read from the board were multiplied by it before any further processing. A loudspeaker (Super tweeter ST400 trio, Selenium Pro) was calibrated using a microphone (4939-A-011, Brüel and Kjær; 1/4 free-field microphone, 
A

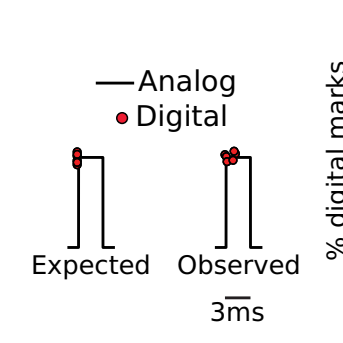

C

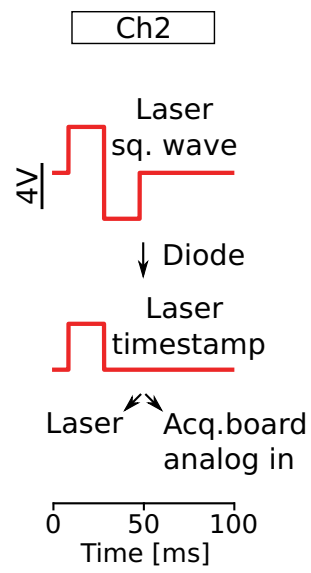

B

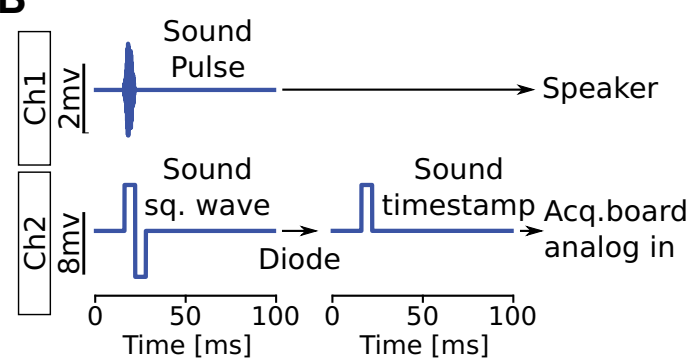

D

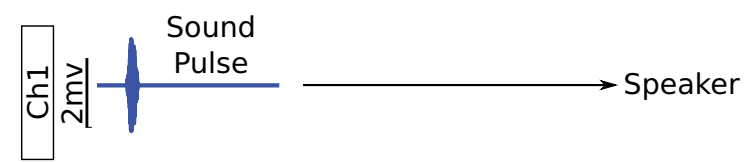

Sound+Laser Sound+Laser

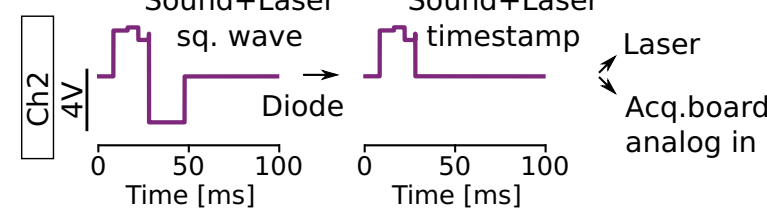

Figure 1. Schematics of sound and light triggers combined with digital time stamps using a two channel sound card. $\boldsymbol{A}$, Schematic showing analog pulses and a small jitter of digital detected timestamps. Right, Histogram showing percentage of recorded pulses and delay to analog edge. $\boldsymbol{B}$, Illustration of channel 1 producing a sound wave to the speaker; channel 2 producing the corresponding square wave with the positive portion with the same duration as the sound pulse (gray shading). Using a diode cuts the negative portion and can be detected as the sound timestamp. $\boldsymbol{C}$, Channel 2 writes the light square wave and the positive portion is split to provide a laser trigger and the acquisition board analog input indicates the light timestamp. $\boldsymbol{D}$, Channel 1 as in $\boldsymbol{B}$, and channel 2 illustrates the sum of light and sound square waves. As in $\mathbf{C}$, both timestamps can be extracted from the signal recorded from the analog input of the acquisition board.

sensitivity of $4.23643 \mathrm{mV} / \mathrm{Pa}) 4.5-10 \mathrm{~cm}$ in front of the speaker. Sound pulses ( $2 \mathrm{~s}$ in duration) were generated at the desired frequency bands with logarithmically decreasing amplification factors (voltage output to the speaker) and simultaneously recorded using a personal computer (HP Z220; Intel Xeon 8-core 3.6 GHz, 16 GB RAM), and the power spectral density (PSD) of the recorded signal was calculated using a Hann window with no overlap. Root mean square (RMS) was calculated as

$$
\sqrt{\sum_{i=1}^{n} P S D_{i} \times \text { BinSize }}
$$

where $P S D$ is a $1 n$ array and BinSize is the spectral resolution. The intensity in decibels sound pressure level (dBSPL) was calculated as

$$
20 \times \log \left(\frac{R M S}{\frac{\text { MicSens }}{2 \times 10^{-6}}}\right),
$$

where MicSens $_{\mathrm{vpa}}$ is the microphone sensitivity in $\mathrm{V} / \mathrm{Pa}$, $0.00423643 \mathrm{~V} / \mathrm{Pa}$ for our microphone. All data were saved to disk and loaded to provide the correct amplification factors for the sound intensity used for sound stimulation. The frequency band generated corresponds to the frequency band of greatest power in the signal spectrum, with border frequencies strongly attenuated. Sound calibrations were routinely repeated before every beginning of an experimental group. The full sound calibration tests 300 amplification factors for the frequency band used for stimulation, providing $0.5 \mathrm{dBSPL}$ precision. Sound stimulation consisted of sound pulses of Gaussian white noise filtered from 5$15 \mathrm{kHz}$, intensity of $80 \mathrm{dBSPL}$ and duration of $3 \mathrm{~ms}$, presented at $10 \mathrm{~Hz}$ ( $3 \mathrm{~ms}$ of sound pulse followed by $97 \mathrm{~ms}$ of silence), repeated for five blocks of 200 pulses.

\section{Light calibration and optogenetic stimulation}

Light intensity calibration was performed before each experiment. Optic fibers of $200 \mu \mathrm{m}$ diameter were cleaned with lens cleaning tissue and ethanol (99.5\%). The light intensity was measured and the laser lens position adjusted until light power at the tip of the fiber was $5-7 \mathrm{~mW} / \mathrm{mm}$ measured by an optical power meter (Thorlabs PM20). Light stimuli triggers were generated and written to the sound card, in which the output was split and connected to the laser input and the data acquisition board (Fig. 1C,D). Light stimulus was delivered using a $473 \mathrm{~nm}$ laser (CNI, 
MBL-III-473; for ChR2) and a 532-nm laser (CNI, MGL-III532; for eArch3.0). Laser stimuli consisted of 200 light pulses at intensity of $5-7 \mathrm{~mW} / \mathrm{mm}$ with $10 \mathrm{~ms}$ duration, presented at $10 \mathrm{~Hz}$ ( $10 \mathrm{~ms}$ on and $90 \mathrm{~ms}$ off) $473 \mathrm{~nm}$ blue light for ChR2 experiments; and a pulse of a total of $20 \mathrm{~s}$ of 543 $\mathrm{nm}$ green light repeated in five blocks with $10 \mathrm{~s}$ interval, so that green light was continuously on during each sound stimulation block for eArch3.0 experiments. For concomitant sound and blue light stimulation, the light pulses were presented $4 \mathrm{~ms}$ before the sound pulses, so that these are embedded in the light pulse.

\section{Digital timestamps marks for sound and light stimulation}

Digital timestamps markers use a combination of a sound card and an Arduino board (Arduino Due; 54 digital pins, 12 12-bit ADCs, two 12-bit DACs), taking advantage of GNU/ Linux audio real-time capabilities. One output of the sound card was connected to the sound amplifier (PM8004, Marantz), and another output to an acquisition board (OpenEphys, Open-ephys.org; v2.2, Opal Kelly XEM6010-LX150, $30 \mathrm{kHz}$ sampling rate, 16 bit, eight ADCs, eight DACs) analog input with a diode as rectifier, conducting only positive voltages. In detail, the two outputs of the sound card are used for sound stimulation (channel 1) and for generating timestamp marks (channel 2). To test the temporal accuracy of the digital input of the acquisition system (Open-Ephys), we recorded 5000 square pulses delivered by both analog and digital inputs to the acquisition board. When compared with analog traces, we found $15 \%$ of the digital timestamps to be delayed by $>150 \mu \mathrm{s}$, which is a jitter of $5 \%$ of the 3ms pulse width (Fig. 1A). To avoid jitter, analog square waves to mark stimulation timestamps were used. To avoid producing capacitive-like traces when using square pulses in a sound card, we used square waves of twice the stimulus duration, containing both positive and negative portions (Fig. 1B). In practice, channels carrying square waves are connected to a diode before connecting to the analog input of the acquisition board or to the laser, thereby only conducting the positive values (Fig. 1B,C). The resultant square waves have the same duration as the stimulus, since only the positive half is conducted (Fig. 1B), thereby channel 2 was used both as timestamp marker and as a trigger for light stimulation.

For experiments using sound synchronized with light stimulation three outputs would be required (one carrying the sound signal to a speaker, one carrying the sound square waves/timestamps and the third carrying square waves for light trigger/timestamps). Here, a square wave of twice the length for light stimulation was used, so when simultaneous sound and light stimulation is required, the sound pulse is written to channel 1 while the sum of the sound and light square waves is written to channel 2 (Fig. 1D). Thereby channel 2 triggers the laser (amplitude $>3.3 \mathrm{~V}$ ), as well as provides edges for timestamp detection.

\section{In vivo units recording}

Animals were anesthetized with ketamine-xylazine (90/ $6 \mathrm{mg} / \mathrm{kg}$, i.p.) and an additional injection of ketamine
$(45 \mathrm{mg} / \mathrm{kg})$ if surgery required. The anesthetized mouse was placed on an electric thermal pad $\left(37^{\circ} \mathrm{C}\right)$ and fixed into a stereotaxic frame with ear bars holding in front of and slightly above ears, on the temporal bone, to not block the ear canals. The skin over the vertex was removed and hydrogen peroxide (3\%) was applied on the skull to visualize sutures. All coordinates were corrected to account for differences in the size of the head of each animal, similar to the virus injection procedure. Next, three small holes were drilled: at $\mathrm{AP}=-6.1 \mathrm{~mm}, \mathrm{ML}=$ $-2.3 \mathrm{~mm}$ (left DCN, for probe placement); at $\mathrm{AP}=-6.1$ $\mathrm{mm}, \mathrm{ML}=2 \mathrm{~mm}$ (for optic fiber placement); and at $\mathrm{AP}=-2$ $\mathrm{mm}, \mathrm{ML}=1 \mathrm{~mm}$ (for reference). Next, a micro screw was fixed in the reference coordinate using polymethyl methacrylate. The optic fiber was inserted into the brain using a micromanipulator positioned in a $45^{\circ}$ angle to a depth of $5.58 \mathrm{~mm}$, ending $0.5 \mathrm{~mm}$ away from the DCN. This angle avoids perturbing auditory pathways and gives appropriate space for the insertion of the recording electrode (NeuroNexus; single shank, 16 channels, $50 \mu \mathrm{m}$ channel spacing, $177 \mu \mathrm{m}^{2}$ recording site, $5 \mathrm{~mm}$ length). The recording electrode was dipped in fluorescent dye (1,1-dioctadecyl-3,3,3',3'-tetra-methylindocarbocyanine perchlorate; Dil; Invitrogen) for $10 \mathrm{~min}$ before the procedure to visualize electrode placement post hoc. Three different recording depths were used (electrode tip at DV $=-4.0$ $\mathrm{mm},-4.3 \mathrm{~mm}$, or $-4.5 \mathrm{~mm})$. Unit responses were recorded under sound and/or light stimulation, with modalities presented at randomized order. Data acquisition was done using a headstage (Intan RHA2116 or Intan RHD2132; 16 unipolar channels) connected to a data acquisition board (Intan RHA2000 or Open-Ephys), at a sampling rate of $25 \mathrm{kHz}$ (for experiments with Intan $\mathrm{RHA}$ ) or $30 \mathrm{kHz}$ (for experiments with Open-Ephys). The headstage reference and ground were separated in the headstage, then the ground was connected to the system ground and the reference was connected to the reference screw. Responses were visualized using Open-Ephys graphical user interface (Siegle et al., 2017). At the end of recordings animals were killed for histology.

\section{Unit analysis}

Spikes were detected and clustered with a fourth order Butterworth digital bandpass filter $(300-7500 \mathrm{~Hz})$, negative spikes detected using a threshold from 2 to 4.5 SD, waveforms of $2 \mathrm{~ms}$ around the detected negative peak, and three features per channel. Peristimulus-time histograms (PSTHs) were calculated by counting occurrence of spikes in a time window of $100 \mathrm{~ms}$ around each transistor-transistor logic (TTL) $(50 \mathrm{~ms}$ before and $50 \mathrm{~ms}$ after the TTL) and presented as mean number of spikes per time, where each bin corresponds to $2 \mathrm{~ms}$. Units were classified as responding units as described by Parras et al. (2017). In brief, 1000 or 2000 PSTHs (depending on the number of trials played) were generated with random values in a Poisson distribution, with $\lambda$ equal to the mean of values from the negative portion of the unit PSTH (baseline). Then, for real and simulated PSTHs, the mean of the negative PSTH values (baseline) was subtracted from the mean of the positive PSTH values (response), resulting in 


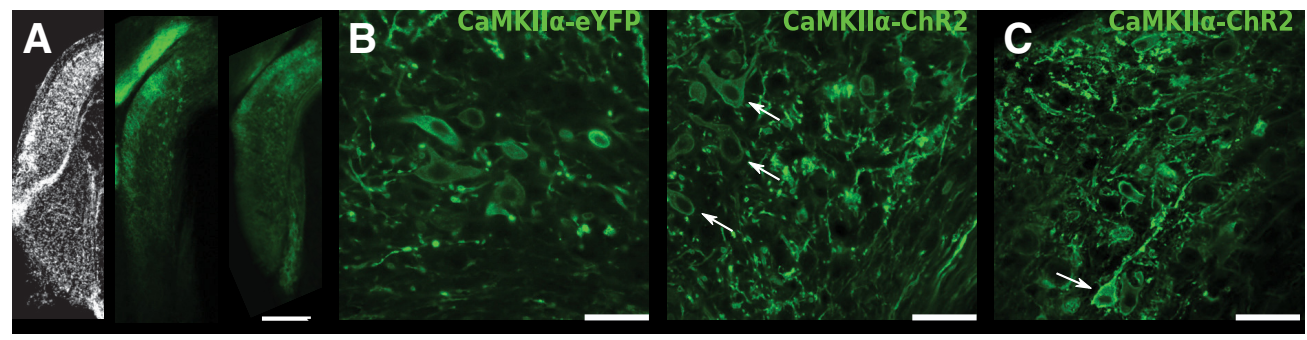

D
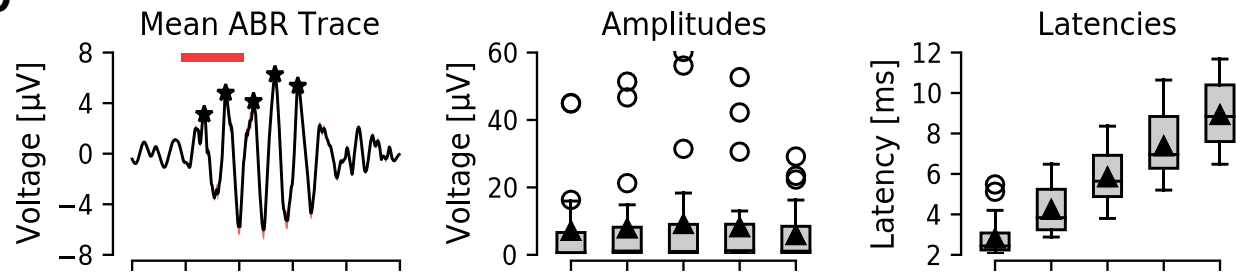

$\mathbf{E}$
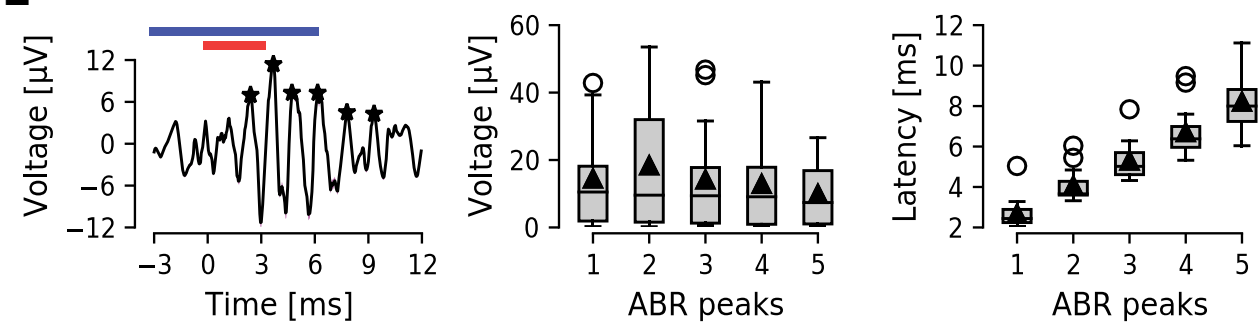

Figure 2. CaMKII $\alpha$-ChR2-eYFP-positive neurons in the DCN and ABR in injected animals. $\boldsymbol{A}$, Image of coronal brainstem sections with the DCN and VCN highlighted after DAPI nuclear staining (left), control CaMKIl $\alpha$-eYFP (center), and CaMKIl $\alpha$-ChR2-eYFP expression (right). Scale bar: $250 \mu \mathrm{m}$. B. High-magnification confocal images showing several elongated horizontal somas (white arrows, possibly fusiform cells) labeled with membrane expression of eYFP. Scale bar: $40 \mu \mathrm{m}$. $\boldsymbol{C}$, Another high-magnification example of CaMKII $\alpha$-ChR2-eYFP labeling of the DCN. Two possible giant cells are in the deep layer (white arrow). Lateral is left and ventral is down for all images. Scale bar: $40 \mu \mathrm{m}$. $\boldsymbol{D}, \boldsymbol{E}, \mathrm{ABR}$ waveforms recorded using electrodes lowered into the DCN in response to sound $(\boldsymbol{D})$ and sound+light $(\boldsymbol{E})$ stimulation protocols. Left, Mean (black line) and SEM (red shadow) ABR traces $(n=13)$, with detected peaks marked with black asterisks. Center, Box plots show group amplitude of the first five ABR peaks, horizontal lines show the median, triangles show mean, circles show outliers, whiskers bounding $99 \%$ of the data points. Right, Group latency of the first five ABR peaks.

a baseline-corrected spike count. Finally, the $p$ value was calculated as

$$
p=(g+1) /(N+1)
$$

where $g$ is the number of simulated histograms with corrected spike count bigger than the real unit spike count, and $N$ is the total number of simulated histograms, which here is the number of trials presented at that unit recording. A cell was classified as responding to a stimulation if the resulting $p$ value was $<0.05$. Cells were classified as responsive to sound only, light only, sound+light only, or sound and light. Spike rate was calculated as spike events per second along all the recording (including the stimulation period). The threshold of $9 \mathrm{~Hz}$ was considered to separate between slow-spiking and fast-spiking neurons, since $\sim 88 \%$ of neurons had firing rate $<6.42 \mathrm{~Hz}$ and the remaining $\sim 12 \%$ had firing rate $>9.24 \mathrm{~Hz}$. Student's $t$ test, two-tailed, unequal variance was applied to compare firing rate between neurons and $p$ values were Bonferroni-corrected when the same dataset was used for multiple comparisons. All firing rate values are represented as frequency \pm SEM.

\section{Auditory brainstem responses (ABRs)}

ABRs are high-frequency event-related potentials that are commonly recorded from surface electrodes placed over the skin of the head or subdermally over the skull, but can also be recorded from auditory central areas (Lev and Sohmer, 1972; Achor and Starr, 1980), or even from brain areas unrelated to auditory processing (Jewett, 1970). Here, ABRs were extracted from the intracerebral electrode for recording extracellular spikes in the DCN. The high-frequency event-related local field potential was filtered (fourth order Butterworth digital bandpass filter from 500 to $1500 \mathrm{~Hz}$ ), sliced ( $3 \mathrm{~ms}$ before and $12 \mathrm{~ms}$ after each sound pulse) and averaged, and ABR peaks were detected as a positive value 1 SD above the mean, larger than the previous value, and larger or equal to the next value. ABR peak values and latencies were then grouped for sound or light stimulation (see Fig. 2).

\section{Histology}

Intracardial perfusion was conducted by deeply anesthetizing mice with ketamine/xylazine $(180 / 12 \mathrm{mg} / \mathrm{kg})$. Animals were fixed in a polystyrene plate, and a horizontal 
incision was made in the skin at the level of the diaphragm. Thoracic cavity was open by cutting the ribs laterally and the sternal medially. A 30G needle was inserted into the left ventricle for perfusion with cold PBS and an incision was made in the right atrium to allow for out-flow. In total, $20-30 \mathrm{ml}$ of cold PBS followed by $20-30 \mathrm{ml}$ of fixative (4\% paraformaldehyde in $0.1 \mathrm{~m}$ phosphate buffer; $\mathrm{pH} 7.4$ ) was used. Next, the brain was dissected and stored in $4 \%$ paraformaldehyde overnight. For free-floating vibratome (OTS-4000, EMS, Hatfield) sections the brain was stored in PBS before slicing; and for cryostat sections, the brain was kept in PBS with $30 \%$ sucrose until dehydrated (visualized by the brain sinking to the bottom of the solution), and frozen using isopentane at $-60^{\circ} \mathrm{C}$. Coronal sections $(120 \mu \mathrm{m}$ thick for freefloating, $30 \mu \mathrm{m}$ for cryostat sections) of the brainstem, containing the DCN, were collected on glass slides and kept dark until examination of fluorescent expression by neurons. Cell nuclei were stained with 4',6-diamidino-2-phenylindole (DAPI; Sigma) to visualize cell layers and borders of the DCN and VCN. Expression of optogenetic proteins was visualized by detection of genetically expressed enhanced yellow fluorescent protein (eYFP). Images were collected using Zeiss Observer Z1 fluorescence microscope or a Zeiss Examiner $\mathrm{Z} 1$ confocal microscope. The objectives N-Achroplan $5 \times /$

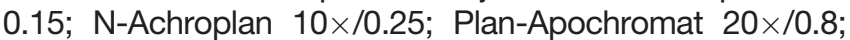
and Plan-Neochromat $40 \times / 0.75$ were used.

\section{CLARITY}

The CLARITY procedure followed standard protocol and was previously described for another brain region (Hilscher et al., 2017). Data from the auditory brainstem was collected during the same experiment as previously published (Hilscher et al., 2017).

\section{Software availability}

Our optogenetic and sound stimulation uses open systems and free open-source software. Recordings were done using Open-Ephys graphical user interface (Siegle et al., 2017). Calculations were done using Scipy (Jones et al., 2001) and Numpy (Van Der Walt et al., 2011), and all plots were produced using Matplotlib v2.2.4 (Hunter, 2007; Caswell et al., 2019). Spikes were detected and clustered using Klusta, and visual inspection was performed using Phy (Rossant et al., 2016). All scripts used for stimulation control and data analysis are available at https://gitlab. $\mathrm{com} / \mathrm{malfatti} / \mathrm{SciScripts}$. Histology images were collected using AxioVision and Zen software, and edited for brightness and contrast in ImageJ (Schneider et al., 2012).

\section{Data availability}

The datasets generated and/or analyzed in the current study are available on request.

\section{Results}

\section{ABRs can be extracted during optogenetic excitation of CaMKIl $\alpha$-ChR2-positive DCN neurons}

We first tested whether the CaMKIl $\alpha$ promoter can be used to control subpopulations of DCN neurons. We injected viral vectors (rAAV5/CamK2-hChR(H134R)-eYFP or control rAAV5/CaMKIla-eYFP) into the DCN for expression of ChR2 and/or eYFP under the control of the CaMKIl $\alpha$ promoter of one- to two-month-old C57BI/6J mice. Four weeks later local protein expression in the DCN was examined, and showed strong eYFP signal in the vicinity of the injection site with a spread to both superficial and deeper layers of the DCN for both control and ChR2 vectors (Fig. 2A, center and right, respectively). eYFP showed strong membrane expression of soma and neurites of DCN neurons for both ChR2 and control constructs, especially in cells with elongated somas perpendicular to the DCN edge with thick basal dendrites spreading toward the molecular layer (possible fusiform cells; Fig. 2B, arrows). Smaller neuronal somas were also labeled with eYFP in the fusiform and deeper layers, as well as several large neuronal somas of the deep layer of the DCN with dendrites stretching along the internal edge of the DCN (possible giant cells; Fig. $2 C$, arrow). This shows that the CaMKII $\alpha$ promoter is not specific for the DCN excitatory fusiform cell layer, although it seems to indeed label excitatory fusiform-like cells and giant cells with strong membrane expression.

To confirm that animals can hear the provided sound stimulation after injection of viral constructs we routinely extracted $A B R$ waveforms from extracellular recordings. A high-impedance electrode (16 channel single shank silicon probe, Neuronexus), placed in the DCN for recording unit responses in anesthetized mice, was used to isolate ABRs in response to sound or concomitant sound and optogenetic stimulation. The anatomic correlation between auditory brainstem structures and ABR peaks, where I corresponds to the auditory nerve, II to cochlear nuclei, III to superior olivary complex, and IV and V to inferior colliculus (Henry, 1979) is useful for verifying an intact auditory brainstem system. We found all animals to display at least five peaks in the ABR waveforms $(n=13)$ in response to $80 \mathrm{dBSPL}$ stimulation (Fig. 2D). Also, ABR mean amplitude and latency was not affected by concomitant sound and light stimulation (Fig. 2E). Together, this shows that the animals can hear the provided sound stimulus after viral vector injection procedure and during concomitant blue light stimulation.

\section{Optogenetic excitation of CaMKIl $\alpha$-ChR2-positive DCN neurons is decreased by concomitant sound stimulation}

Next, we wanted to examine how DCN units respond to optogenetic modulations using the CaMKII $\alpha$ promoter for expression of ChR2 within the DCN. Mice previously injected with CaMKII $\alpha$-ChR2 were anesthetized and fitted to a stereotaxic frame and a silicone 16-channel electrode was lowered vertically into the DCN (Fig. $3 A$ ). A total of 224 isolated units were identified, of which 148 were excluded for not responding to neither sound nor light stimulus (Table 1). From the remaining 76 units $(n=8$ mice) $71 \%(54 / 76)$ responded to sound stimulation $(3 \mathrm{~ms}$, $80 \mathrm{~dB}, 5-15 \mathrm{kHz}$ noise pulses presented at $10 \mathrm{~Hz}$ ) and response to sound was quantified and visualized by PSTHs 

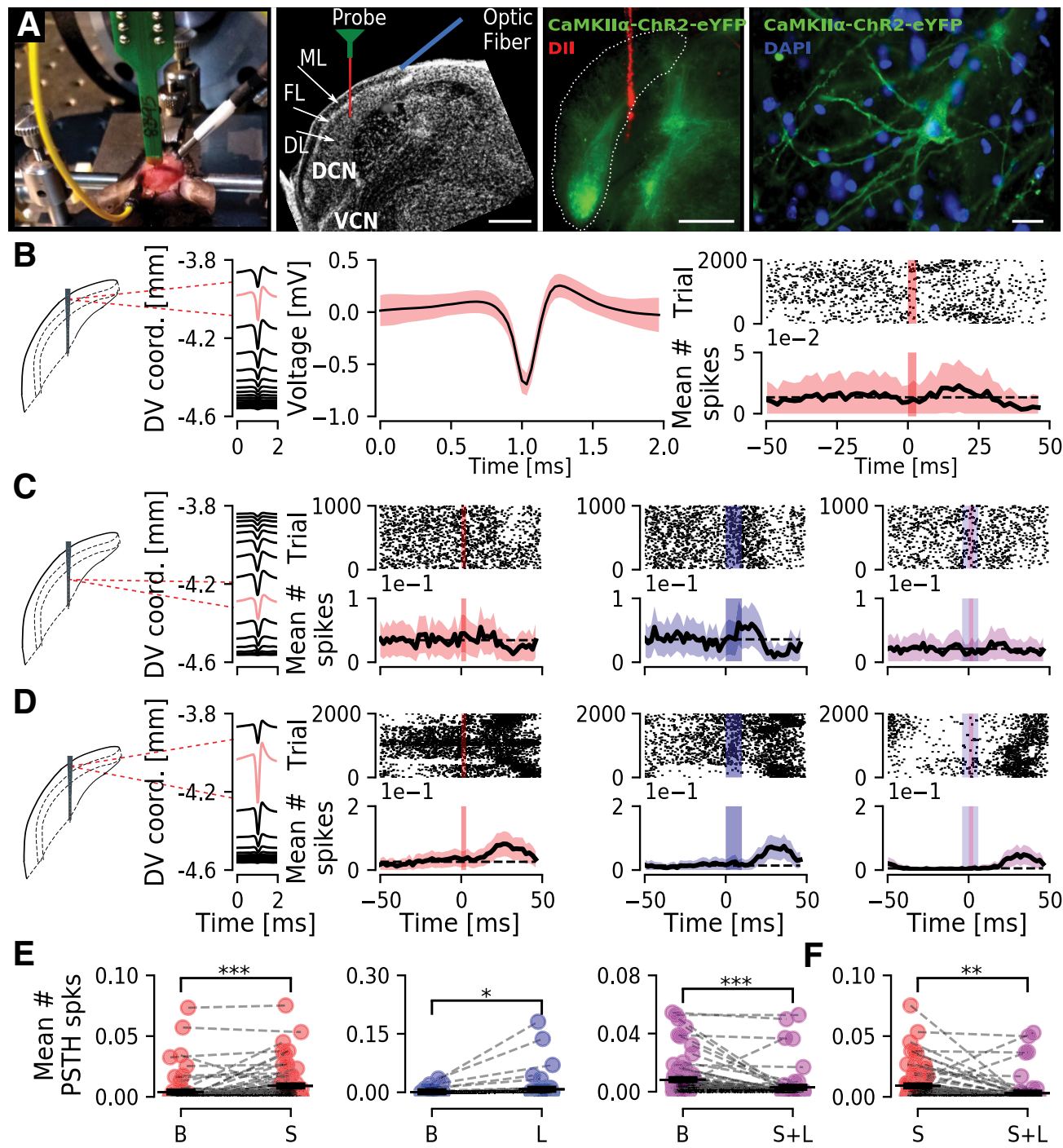

Figure 3. Activation of CaMKII $\alpha$-ChR2-eYFP expressing neurons in the DCN during sound stimulation can decrease unit firing. $\boldsymbol{A}$, left, Photography of a mouse in the recording setup and schematic representation of recording electrode and optic fiber for blue light stimulation in a DAPI labeled section showing the DCN sub-regions (ML-molecular layer, FL-fusiform cell layer, DL-deep layer). Center, Confocal image showing an example of eYFP expression in the dorsal region of the DCN with the probe tract colored by Dil. Scale bar: $100 \mu \mathrm{m}$. Right, Example of a DCN neuron expressing eYFP along the somatic membrane and proximal dendrites. Scale bar: $20 \mu \mathrm{m}$. B , Example of a superficial unit (DV depth in $\mathrm{mm}$ ) with its waveform shown, mean (black line), SEM (red shadow) at higher magnification (center), that responded to sound stimulation (red vertical bar) by increasing firing rate as seen by a PSTH. $\boldsymbol{C}$, Example of a deeper unit that does not respond to sound stimulation (left), increases firing in response to blue light stimulation (blue vertical bar, center), but shows no increase on both light and sound stimulation (right). $\boldsymbol{D}$, Example of a unit responding to sound (left), blue light (center) but with a slight decrease to concomitant sound and light stimulation (right). E, Group mean number of spikes for all units $(n=76)$ showing a significant increase after sound (S) comparing to baseline (B; left) or blue light ( $L$; center) stimulation ( $p=5.3 \mathrm{e}-5$ and 0.016$)$ and a significant decrease after concomitant sound and light stimulation ( $\mathrm{S}+\mathrm{L}$; right; $p=8.8 \mathrm{e}-5)$. $\boldsymbol{F}$, Group mean number of spikes for all units $(n=76)$ after sound is higher than after sound+light stimulation $(p=5.3 e-4)$. ${ }^{*} p<0.05 ;{ }^{* *} p<0.005 ;{ }^{* * *} p<0.0005$.

(Fig. 3B,E). Blue light stimulation $(473 \mathrm{~nm}, 10 \mathrm{~ms}$ in duration, at $10 \mathrm{~Hz}$ with intensity of $5-7 \mathrm{~mW} / \mathrm{mm}^{2}$ at fiber tip) delivered by a glass optic fiber to the DCN $(\varnothing 200 \mu \mathrm{m}$, inserted in a $45^{\circ}$ angle from the contralateral side; Fig. $3 A$ ), elicited increased firing of units immediately following blue light stimulation (Fig. 3C,E). We found 25\% (19/76) units responding to light stimulation. Out of these, $58 \%$ (11/19) responded exclusively to light stimulation, 26\% (5/ 19) responded to either sound or light stimulation (Fig.
$3 D)$, and $16 \%(3 / 19)$ responded only to a combination of sound and light stimuli.

The majority of units, $86 \%(65 / 76)$, had firing rates from $0.05 \sim 6.46 \mathrm{~Hz}$ (average $1.5 \pm 0.19$ ) while a smaller proportion of units recorded had higher firing rates $(14 \% ; 11 / 76)$, from $8.83 \sim 72.43 \mathrm{~Hz}$ (average $26.76 \pm 5.81 \mathrm{~Hz}$; Table 1). Including all 76 responding units, averaged over $1.5 \mathrm{~min}$ including stimulus epochs, and comparing to specific stimuli showed a significant increase in response to 
Table 1: Number of recorded units, separated by group, responding (sound and/or light stimuli), firing rate (low $<\mathbf{9 H z}$, high $>9 \mathrm{~Hz}$ ), and direction of modulation (down arrow decrease and up arrow increase firing rate on concomitant sound and light stimulation comparing to sound alone)

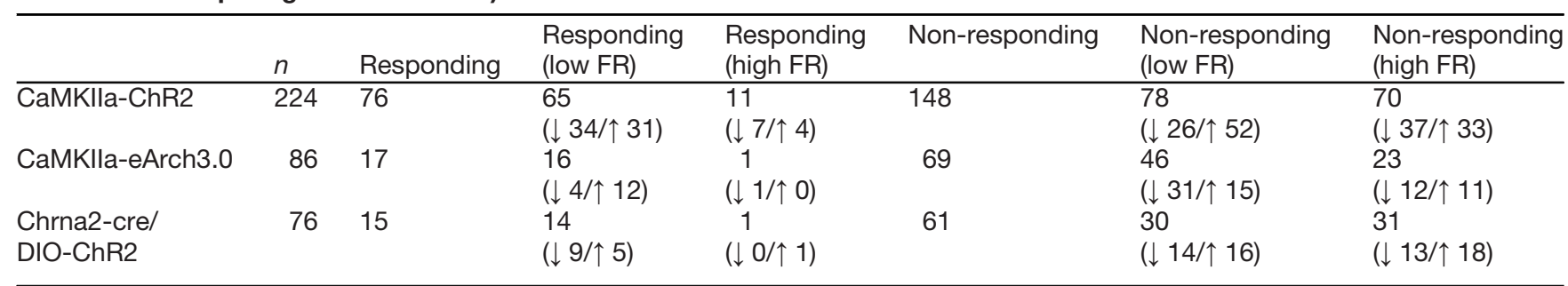

sound $(p=5.3 e-5$; Fig. $3 E$, left) or light $(p=0.016$; Fig. $3 E$, center) but, interestingly, a decrease in response to concomitant sound and light stimulation $(p=8.8 \mathrm{e}-5$; Fig. $3 E$, right). Also, the mean number of spikes in response to sound were significantly higher than to concomitant sound and light $(p=5.3 e-4$; Fig. $3 F)$. This shows that optogenetic excitation using $\mathrm{ChR} 2$ can increase firing of DCN units, even in units not directly responding to sound or light, but controversially, presence of simple sounds during optogenetic stimulation can decrease the overall unit firing.

\section{Inhibiting CaMKIl $\alpha$-eArch3.0-positive neurons in the DCN delay response to sound and bidirectionally affect units not responding to sound}

We then tested whether we could inhibit CaMKII $\alpha$ eArch3.0-positive DCN neuron response to sound using the outward proton pump eArch3.0. We injected adult (one month) wild-type C57BI/6J mice unilaterally with Archaerhodopsin-containing viral vectors (CaMKII $\alpha$-eArch3.0eYFP) and four weeks later extracellular activity in the DCN was recorded. Units were recorded in response to short sound pulses $(3 \mathrm{~ms}, \quad 80 \mathrm{~dB}, 5 \sim 15-\mathrm{kHz}$ noise pulses presented at $10 \mathrm{~Hz}$ ) and next using an optic fiber coupled to a green laser source $(543 \mathrm{~nm}$ excitation) we examined whether responses to sound could be abolished by concurrent green light stimulation $(543 \mathrm{~nm}, 20 \mathrm{~s}$, repeated $5 \times$ with $10 \mathrm{~s}$ interval, so it is concomitant to sound pulses). Out of 86 units isolated ( $n=4$ mice), we found that 17/86 (20\%) units responded to stimulation (Fig. $4 D$, left), from which 12/17 (71\%) responded to sound stimulation (Fig. $4 E$, left). The most striking finding was that instead of inhibition of responses to sound we found several types of unit responses being delayed during green light stimulation (Fig. 4A,B); $7 / 17(41 \%)$ units that sharply responded to sound showed a delayed response to sound with mean latency of $19.1 \pm 1.22 \mathrm{~ms}$ when the DCN CaMKII $\alpha$-eArch3.0 cells were inhibited during sound stimulation (Fig. 4A-C,E, right). The delayed response to sound continued strongly time-locked. This could suggest that some DCN CaMKII $\alpha$ eArch3.0-positive neurons may be inhibitory, as green light stimulation could involve disinhibition of cartwheel cells, and complex spiking units are known to generate delayed responses in PSTH in the range of 10 to $40 \mathrm{~ms}$ delay (Parham et al., 2000). Examining responses from the units isolated, the majority (10/12) of units responded to sound stimulation by increasing firing rate (Fig. $4 A, B$ ), while 2/12 units decreased the firing rate in response to sound stimulation (Fig. 4C) and both such responses were delayed in the presence of green light stimulation. Furthermore, 5/17 units responded exclusively to sound, while another $5 / 17$ units responded exclusively to the combination of sound and green light (Fig. 4D, right).

Examining firing rates of sound responding units and comparing to non-responding units showed that we targeted slow firing units responding to sound (1.5 \pm 0.6 and $9.1 \pm 2 \mathrm{~Hz}$ for responding and non-responding units, respectively). Still, it is also important to examine the effect of DCN inhibition on units not responding directly to sound. We found, for the remaining 69/86 units that did not respond to sound stimulation, 39\% (27/69) of units to decrease spontaneous firing rate under green light stimulation (Fig. $5 A, C$ ). Out of the 27 units, 12 were high-frequency firing $(33.51 \pm 6.3 \mathrm{~Hz})$ that decreased firing to $70 \%$ of the initial frequency $(23 \pm 6.68 \mathrm{~Hz})$ under green light stimulation, while 15 units were low-frequency firing $(2.19 \pm 0.57 \mathrm{~Hz})$, decreased firing frequency by $58 \%$ $(1.26 \pm 0.48 \mathrm{~Hz})$ on green light stimulation. On the contrary, 42 units increased firing frequency on green light stimulation (Fig. 5B,C), where 11 high-frequency firing units increase in firing frequency to $\sim$ double the initial frequency $(16.4 \pm 4.38$ to $34.11 \pm 6.76 \mathrm{~Hz})$ while 31 low firing units on average increased firing from $0.33 \pm 0.11$ to $1.54 \pm 0.29 \mathrm{~Hz}$ (Fig. 5B,C). Overall, non-responding units that had low firing rates in response to sound showed a significant increase in firing rate ( $n=46 / 69$ units, $p=0.03$ ). Also, CaMKII $\alpha$-eArch3.0 inhibition caused a bidirectional effect, with 27/69 decreasing $(p=4 e-4)$ and 42/69 increasing $(p=0.01)$ firing rate (Fig. $5 C)$. This highlights the complexity of the DCN and how precaution must be taken when attempting to decrease neuronal activity in vivo of the auditory brainstem using tools such as CaMKII $\alpha$ eArch3.0.

\section{The Chrna2-cre transgenic line targets putative T- stellate cells and bushy cells of the VCN}

A recent and interesting transgenic cre-line is the chrna2-cre mouse that targets specific interneuron populations in different brain and spinal cord regions (Leão et al., 2012; Mikulovic et al., 2015; Perry et al., 2015; Hilscher et al., 2017; Siwani et al., 2018). So far there are no reports of Chrna2-cre expression in auditory areas, so here we start by exploring Chrna2-cre expression in the cochlear nucleus and superior olivary complex. Heterozygous Chrna2- 
A

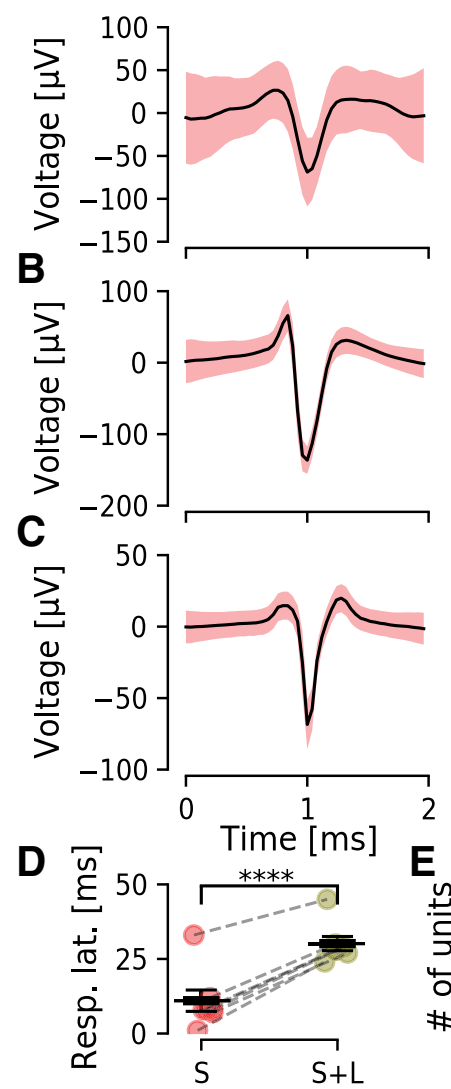

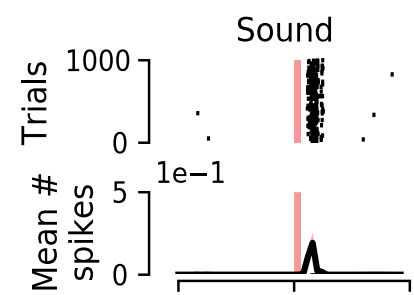

$\left.\begin{array}{cc}\frac{n}{\sigma} & 1000 \\ \hdashline & \\ \models & 0\end{array}\right]$
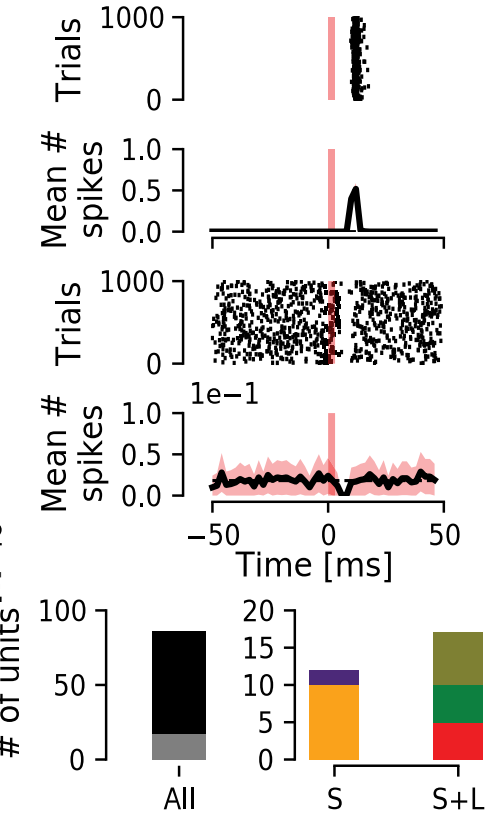
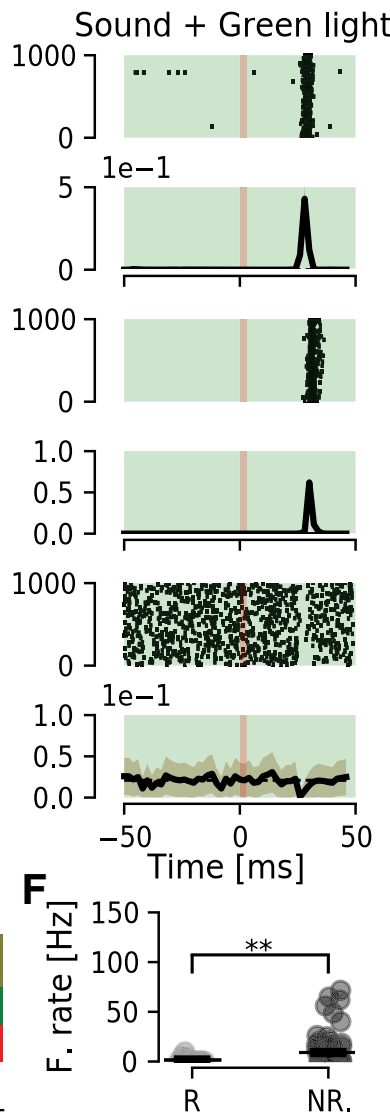

Figure 4. Inhibition of CaMKII $\alpha$-eArch3.0 expressing neurons can delay unit sound responses. $\boldsymbol{A}$, Example of a unit (mean, black line; SEM, red shadow) that responds to sound, while during inhibition of CaMKIl $\alpha$-eArch3.0-positive DCN cells using green light (5to $7-\mathrm{mW} / \mathrm{mm}^{2}$ laser intensity, green background) this unit shows delayed excitation in response to sound. $\boldsymbol{B}$, Another unit showing time-locked excitation in response to sound stimulation. This excitation is delayed by $\sim 20$ ms when CaMKIl $\alpha$-eArch3.0-positive DCN cells are inhibited by green light. $\boldsymbol{C}$, Example of a DCN unit with a negative response following sound stimulation. This pause in firing is delayed by CaMKII $\alpha$-eArch3.0-positive DCN cells inhibition using green light. $\boldsymbol{D}$, Group latency in response to sound is significantly increased for all units responding to both stimulation $(n=7, p=6.7 \mathrm{e}-6)$. $\boldsymbol{E}$, Quantification of numbers of units. Left, Out of 86 units (black), 17 (gray; 20\%) responded to the provided stimulation. Right, Out of 12 units responding to sound, 10 (orange; $83 \%$ ) increased and two (purple; 17\%) decreased firing in response to sound. Out of 17 responding units, five (red, 29\%) responded only to sound (S), five (green, 29\%) responded only to sound and light combined (S+L), and seven (olive, 41\%) responded to both stimulations. $\boldsymbol{F}$, Responding (R) units showed lower firing rate than non-responding (NR) units $(p=5.8 \mathrm{e}-4)$. ${ }^{* \star} p<0.005 ;{ }^{\star \star \star \star} p<0.00005$.

cre transgenic mice were crossed with homozygous tdTomato-lox reporter mice to visualize Chrna2+ cells in the auditory brainstem (Figs. 6, 7A). We found Chrna2+ cells in the VCN, with dense projections of axons branching into the DCN as well as to the superior olivary complex (Figs. 6, 7A). Brains processed for CLARITY examination (Hilscher et al., 2017) also show Chrna2 + VCN cells with projections to the DCN (Fig. 6E). In order to identify the boundaries of DCN and VCN and examine any soma labeling in the DCN, slices of Chrna2-tdTomato animals were co-stained with DAPI (Fig. $6 C)$. Very few red labeled somas were identified in the DCN of Chrna2-tdTomato animals or in CLARITY images and did not relate to any particular region (Fig. $6 C, E$ ). Next, Chrna2-cre mice were injected with cre-dependent ChR2 (Chrna2-cre/ DIO-ChR2-eYFP) viral vector into the VCN, and the expression pattern was similar to the Chrna2-tdTomato (Fig. 6D). Based on projection patterns we assume Chrna2+ cells of the VCN to comprise of both stellate and bushy cell subtypes (Fig. 6,
$7 A)$. Previously, T-stellate cells have been shown to respond to cholinergic agonists (have acetylcholine receptors), while Dstellate cells are insensitive to carbachol (Fujino and Oertel, 2001); therefore, Chrna2-cre-positive neurons projecting to the DCN (Fig. 6E) are most likely T-stellate cells (Oertel et al., 2011). T-stellate cells also project to the ipsilateral LSO (Oertel et al., 2011), which supports the strong labeling of ChR2 in the ipsilateral LSO (Fig. 6D). The ipsilateral LSO is also labeled by VCN bushy cells as projections to the contralateral medial nucleus of the trapezoid body (MNTB; Fig. 6F) as well as the ipsilateral LSO were apparent, thereby indicating that Chrna2-cre labels both globular and spherical bushy cells.

\section{VCN Chrna2+ neurons can generate indirect optogenetic activation of DCN neurons}

To investigate whether optogenetic control of putative T-stellate cells can excite DCN neurons, Chrna2-cre mice 
A

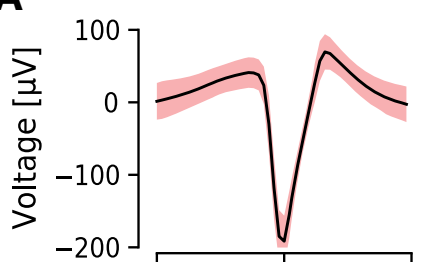

B

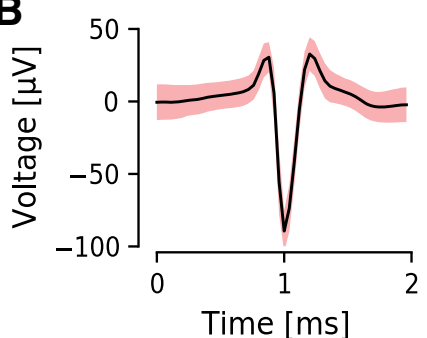

Sound
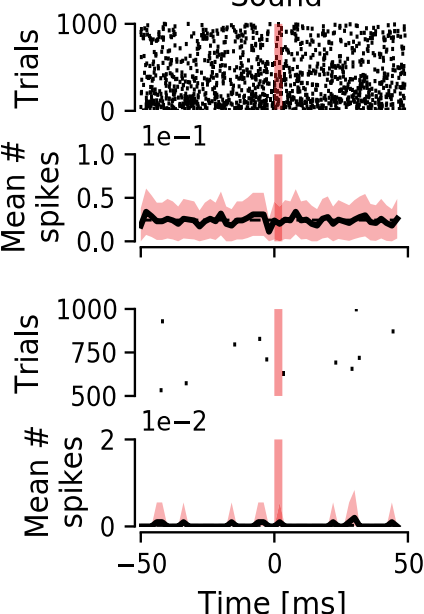

Sound + Green light

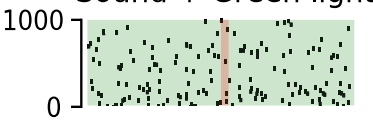

$1 \mathrm{e}-2$

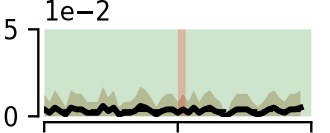

1000

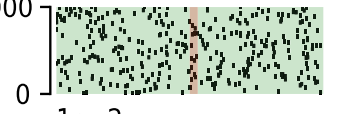

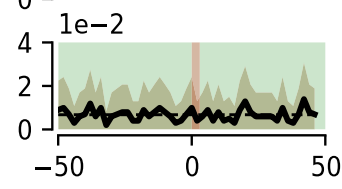

Time [ms]
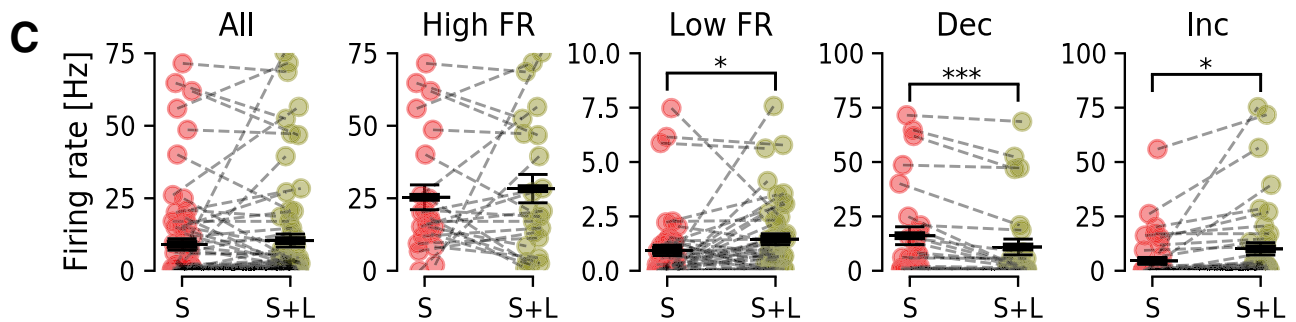

Figure 5. Inhibition of CaMKII $\alpha$-eArch3.0-positive cells in the DCN can both increase and decrease excitation of DCN units not responding directly to sound. $\boldsymbol{A}$, DCN unit (-3.8 mm DV) showing high spontaneous firing but not responding directly to sound (center) showing decreased firing rate on green light stimulation (inhibiting CaMKIl $\alpha$-eArch3.0-positive DCN cells, right). $\boldsymbol{B}$, Unit at similar depth as $\boldsymbol{A}$ showing increased firing rate on inhibition of CaMKIl $\alpha$-eArch3.0-positive DCN cells, but no response directly to sound. $\boldsymbol{C}$, Group firing rate of all units not responding directly to sound ( $n=69)$ in the presence of sound (red) or concomitant sound and green light (olive). Units were divided into high and low firing rate, and a significant increase in firing was found for low firing units $(p=0.03)$. Units were also divided into units that decrease or increase firing rate comparing both stimulations, and a significant decrease and increase was found $\left(p=4.7 \mathrm{e}-4\right.$ and 0.01 , respectively). ${ }^{*} p<0.05 ;{ }^{\star \star \star} p<0.0005$.

were unilaterally injected with cre-dependent ChR2 (Chrna2-cre/DIO-ChR2-eYFP) into the VCN generating labeling of diffuse fibers spreading in the deep layers of the DCN (Fig. 7A). To investigate whether DCN cells can be indirectly excited by light stimulation of ChR2-eYFP expressing Chrna2 + cells of the VCN, we recorded unit responses in the DCN from anesthetized mice while stimulating with blue light $(10 \mathrm{~ms}, 473 \mathrm{~nm}$ light pulses at $10 \mathrm{~Hz}$ ) with an optic fiber placed in a $45^{\circ}$ angle into the VCN. We first analyzed whether units responded to sound stimulation. Out of 76 units extracted, only 15 units $(n=8$, 6 , and 1 , from three mice, respectively) responded to sound and/or light stimulation (Fig. 7B,E). For units not responding to sound, we found examples of a DCN unit with response to VCN light stimulation that was prolonged and appeared increased in the presence of sound (Fig. $7 C)$. Also, one DCN unit with highly temporally precise responses to VCN light showed a loss of response in the presence of sound (Fig. 7D). In summary, 4/15 units responded only to light stimulation, 2/15 units responded only to sound stimulation (Fig. $7 B, E$ ), and $13 / 15$ units responded to light or combined light and sound stimulation (Fig. 7C,E). Excitation of VCN Chrna2+ cells significantly increased firing rate in DCN units, both responding and not responding to sound (Fig. $7 F$ ). Still, the identity of these different units has to be further investigated but highlights that the Chrna2-cre line has potentials for auditory research.

\section{Light stimulation alone does not alter the DCN circuit response to sound}

As recent work has shown that optogenetic light stimulation heats up tissue and many physiological responses are heat sensitive (Owen et al., 2019), it is important to confirm that our findings are not because of local heating of the DCN or VCN. Therefore, we tested both the blue and green light stimulation in control, uninjected mice $(n=5)$. The control experiments $(n=94$ units of which 18 units responded to sound stimulation) showed no significant change in overall number of spikes in response to blue light pulses (Fig. $8 A, B$ ), thereby not affecting the sound responses. Sound pulses during green light stimulation protocols showed no change in mean number of spikes (Fig. $8 A, B$ ) nor changes in firing frequency in response to concomitant $20 \mathrm{~s}$ green light stimulation (Fig. $8 C)$. Also the depth of light penetration is important to take into consideration (Ash et al., 2017) for studying circuit output. Depth profile plots of all recorded units (Fig. $8 D$ ) and of units responding to light (Fig. $8 E$ ) show that 


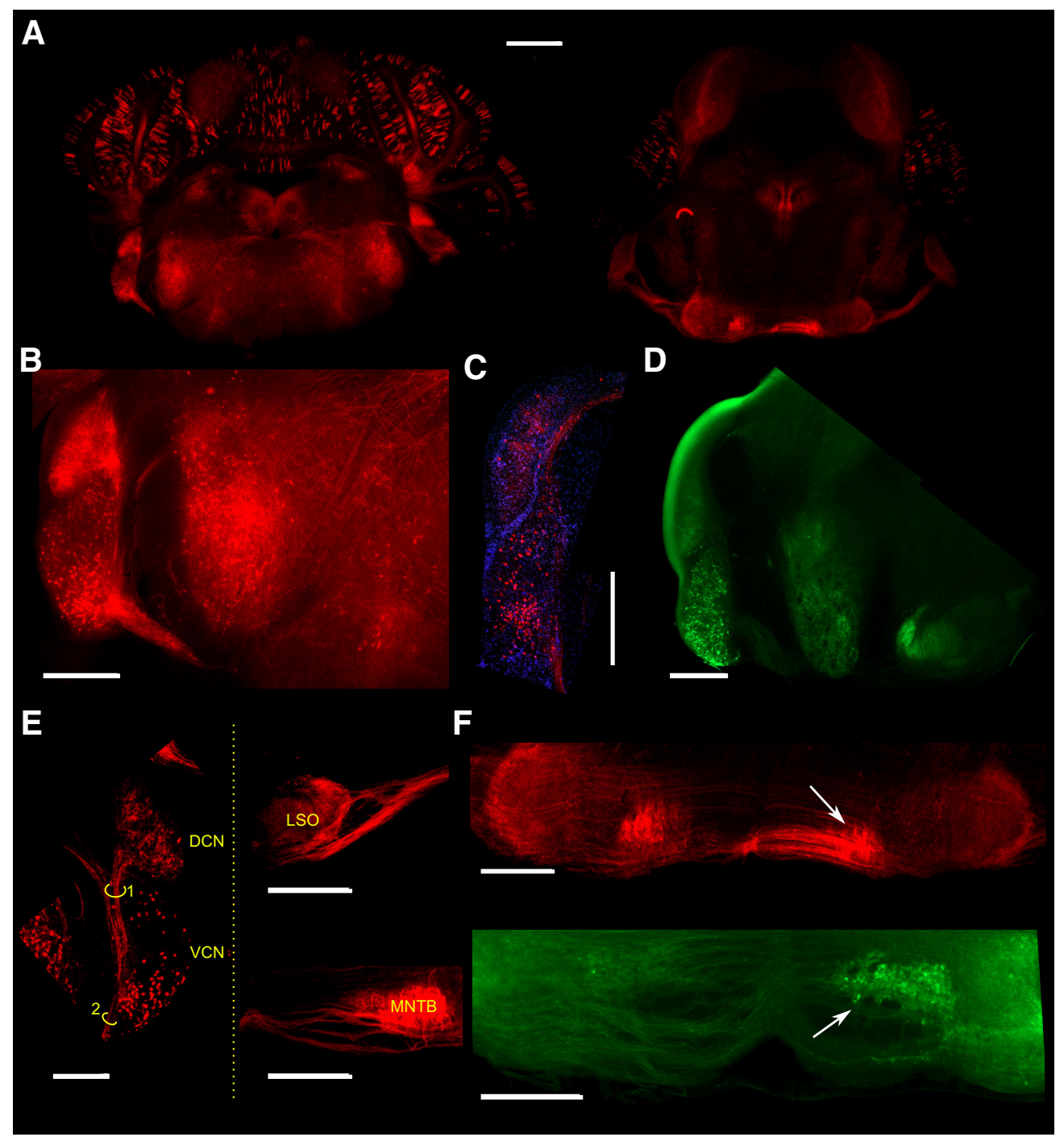

Figure 6. Confocal images showing tdTomato expression and Chrna2-cre/DIO-ChR2-eYFP expression in transversal brainstem sections from Chrna2 ${ }^{\text {cre }} /$ tdTomato $^{\text {lox }}$ mice. A, Mosaic of images showing a coronal overview of Chrna2-tdTomato expression in the cochlear nucleus (left) and MNTB (right) AP coordinate. $\boldsymbol{B}$, Zoom-in image showing tdTomato expression in the VCN and DCN. Red cell bodies can be clearly seen in the VCN area while red expression is more diffuse in the DCN, suggesting this area is showing dense axonal terminations from VCN T-stellate cells. $\boldsymbol{C}$, Another example showing Chrna2-tdTomato expression overlayed with DAPI staining. $\boldsymbol{D}$, Image showing unilateral expression of eYFP following local injections with cre-dependent ChR2 (Chrna2-cre/ DIO-ChR2-eYFP) constructs in the VCN. The VCN contains strongly labeled cell bodies and the DCN shows diffuse green labeling. The strong green edge of the DCN is an artifact of the mounting medium. The ipsilateral S-shaped LSO is also strongly labeled by eYFP. $\boldsymbol{E}$, Image of a coronal slice from the CLARITY dataset of a Chrna2cre ${ }^{\text {lox }}$ mice showing the cell bodies in the VCN and projections going up to the DCN. Highlighted bundles project to LSO (1) and MNTB (2). $\boldsymbol{F}$, top, Zoom-in showing strong labeling of the MNTB and the lateral superior olive (LSO; arrow) suggests that also anteroventral VCN bushy cells are expressing the Chrna2 promoter. Bottom, Subsequent brainstem section (from the same injected animal from $\boldsymbol{D}$ showing strong labeling of the contralateral MNTB (arrow). Scale bars: $1 \mathrm{~mm}(\boldsymbol{A})$ and $500 \mu \mathrm{m}(\boldsymbol{B}-\boldsymbol{F})$.

units recorded responding to light pulses are spread across the DCN DV axis. This suggests that the light penetration was sufficient to reach a large portion of the DCN. Also, this supports the conclusion that different cell types are targeted by the CaMKII $\alpha$ promoter as different cell types of the DCN are organized in different layers. Similarly, Chrna2 + VCN cell light activation, with the fiber targeting the VCN region, triggered activity at different depths of the DV axis of the DCN (Fig. 8E). Thereby the Chrna2+ (putative T-stellate cells) cell axons might target a large area of the DCN as also suggested by CLARITY images.

\section{Discussion}

There is a large variety of genetic tools for dissecting the role of specific neuronal populations, such as transgenic mice expressing a reporter protein or a recombinase, or viral vectors with different promoters for certain cell specificity. Currently widely used viral vector/promoter-based gene expression in neuroscience are hSyn 

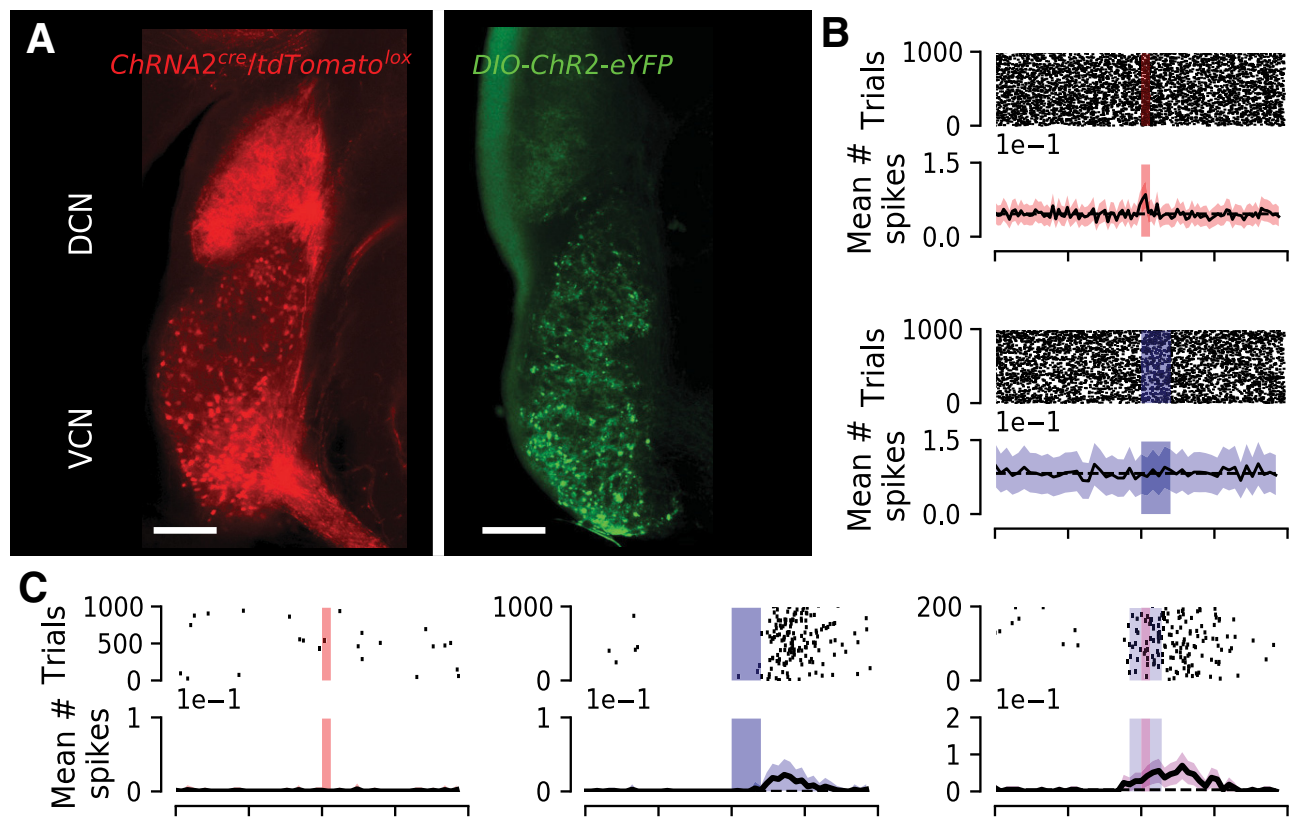

D
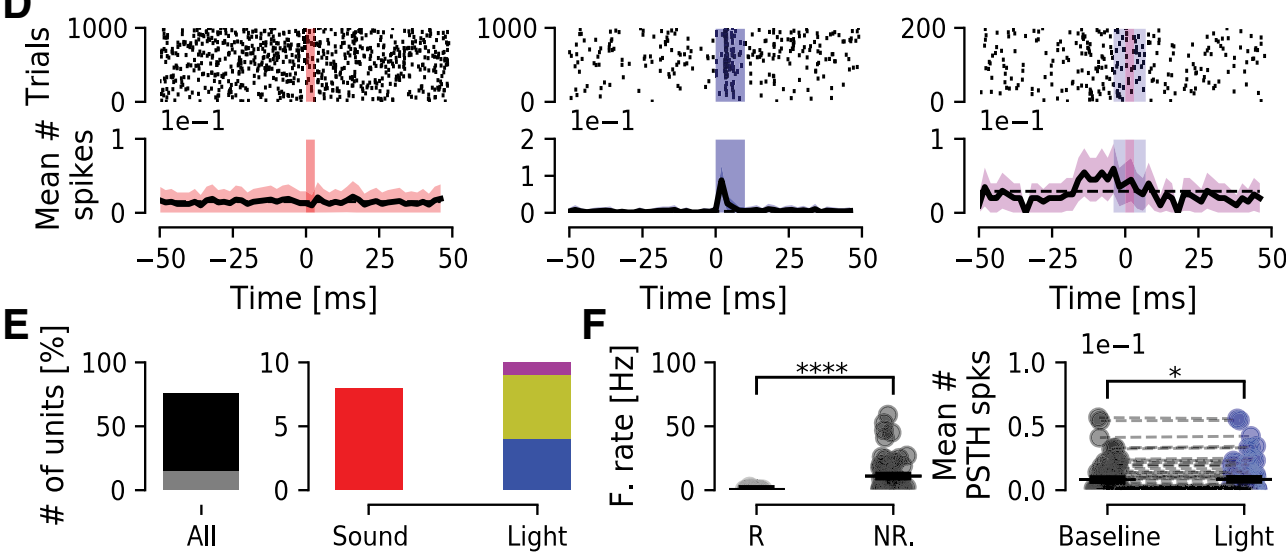

Figure 7. Chrna2-cre-positive neurons of the VCN can be targeted to drive activity of DCN neurons. $\boldsymbol{A}$, left, Confocal image showing tdTomato (red) expression in VCN cell bodies and strong axonal arborizations in the DCN fusiform and deep layers. Right, Expression of ChR2-eYFP (green) in Chrna2-cre-positive neurons of the VCN, with diffuse green innervation of the DCN. B, Example of a unit from the DCN that responds to sound but not light stimulation. Line PSTH bin size is $1 \mathrm{~ms}$ for sound and $2 \mathrm{~ms}$ for light responses. C, Example of a unit that does not respond to sound stimulation, but responds to light stimulation (center). In 200 trials of combined light and sound stimulation the unit responds to both stimuli, with what appears as an anticipation of sound. $\boldsymbol{D}$, Example of a unit not responding to sound pulses but responds with high fidelity to light stimulation of the VCN. When sound and light stimuli were combined, the unit failed to respond. $\boldsymbol{E}$, Quantification of number of units according to response. Left, Out of 76 units, 15 (gray) responded to stimulation. Right, Out of 15 responding units, eight (red; 53\%) responded to sound, four (blue; $27 \%$ ) responded only to light, five (yellow, 33\%) responded to both sound and light, and only one (magenta, $7 \%$ ) responded to light or concomitant sound and light stimulation. $\boldsymbol{F}$, left, Group firing rate of responding (R) units show lower firing than non-responding (NR) units $(p=2.6 \mathrm{e}-7)$. Right, Group mean number of spikes for all DCN units, showing a significant increase after light stimulation of the VCN circuit (black, baseline; blue, light stimulation; $n=76$ units, $p=0.026$ ). ${ }^{\star} p<0.05$; ${ }^{\star * \star *} p<0.00005$.

(synapsin) for all neurons, CaMKII $\alpha$ for cortical pyramidal cells, mDLx for interneurons, GFAP for glia and a cohort of synthetic (any cell) promoters. In regard to transgenic mice (e.g., expressing cre recombinase under the control of specific gene expression), there is a large library of strains for targeting specific interneuron populations in the cortex or single neurotransmitter systems in subcortical nuclei like SERT-cre (serotonergic), DAT-cre (dopaminergic), Chat-cre (cholinergic) etc. However, there is, to our knowledge, no Cre line or promoter-based vector for specific tagging of auditory neurons, especially those residing in the brainstem.

In this work, we first show that the CaMKII $\alpha$ promoter targets DCN neurons of different morphology, and at different depths of the DCN, and thereby not only putative fusiform cells/fusiform cell layer. Next, we show that excitation of CaMKII $\alpha$-ChR2-positive DCN cells during sound stimulation generates normal ABRs, and does not disrupt hearing pathways. Still, we show that optogenetic excitation of CaMKIl $\alpha$-ChR2-positive DCN cells modulates 
A
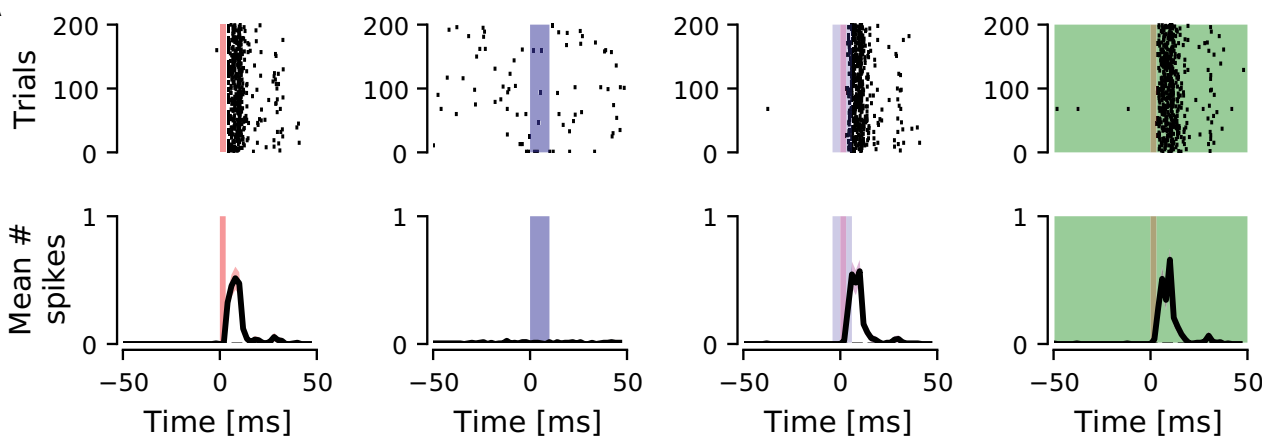

B
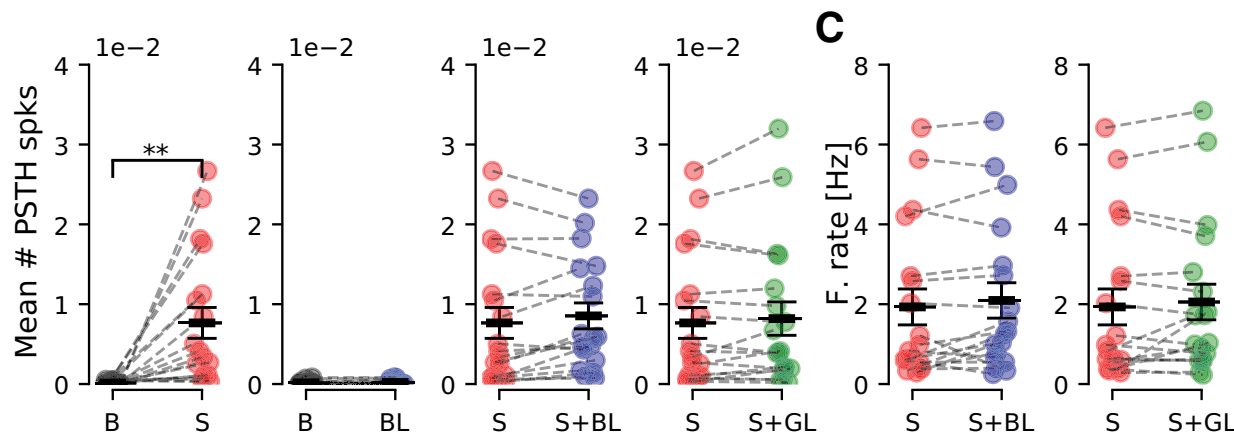

D
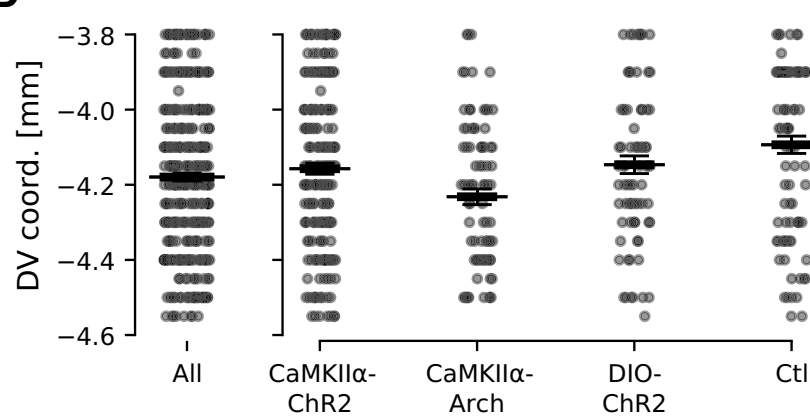

E

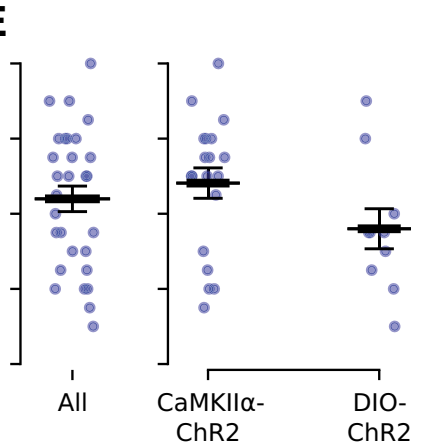

Figure 8. Blue and green light $\left(5-7 \mathrm{~mW} / \mathrm{mm}^{2}\right)$ do not alter DCN unit firing in response to sound in control animals. $\boldsymbol{A}$, Example of a DCN unit showing distinct onset response to sound (left, 200 repetitions shown), no response to blue light pulses (200 pulses, $10 \mathrm{~ms}$ each, presented at $10 \mathrm{~Hz}$ ), and no change in the sound response with concomitant, continuous blue (10 ms) or green light stimulation (20 s). $\boldsymbol{B}$, Group data ( $n=18$ units, 5 animals) showing mean number of PSTH spikes increasing from baseline (B) firing to sound responses ( $\mathrm{S} ; p=4.9 \mathrm{e}-3)$, and no response to blue light simulation (BL, center). Sound responses were not altered by concomitant continuous blue or green light (GL) stimulation. C, Firing rate was not significantly altered by blue or green light stimulation. $\boldsymbol{D}$, DV coordinates for all recorded units from all groups (left) and separated for each group (right). $\boldsymbol{E}$, DV coordinates for all units responding to light stimulation (left), separated for each group (right). The CaMKII $\alpha$-eArch3.0 group was not included, since there was no light-only stimulation. ${ }^{\star *} p<0.005$.

DCN unit firing rate, and that such light stimulation is sensitive to concurrent sound stimulation. When aiming to inhibit DCN activity we found that inhibiting CaMKIl $\alpha$ eArch3.0-positive DCN cells can delay response to sound instead of decreasing DCN units firing rate. More so, in units not responding directly to sound, CaMKII $\alpha$-eArch3.0 inhibition of the circuit could bidirectionally alter firing rate of units. Lastly, we show that the Chrna2-cre line strongly labels cell bodies in the VCN and that these cells are putative T-stellate cells, based on pharmacology and projection patterns (Oertel et al., 2011), and bushy cells, based on innervation to the MNTB and LSO. Furthermore, Chrna2-cre/DIO-ChR2 excitation in the VCN increased firing of a small number of DCN units and this excitation appeared temporally precise. Still, this connectivity needs to be further characterized both presynaptically and postsynaptically.

Our experiments were performed in C57BL/6J mice as these animals are commonly used for genetic manipulations with optogenetic tools. It is known that the C57 mouse strain has a point mutation in the cdh23 gene (Noben-Trauth et al., 2003) and thereby suffers a progressive high-frequency hearing loss after approximately three months of age. Therefore, we performed all experiments in mice around two months of age, with viral constructs injected at one month of age and allowed three to 
four weeks for adequate protein expression before optogenetic experiments. Still, it is important to confirm that animals indeed can hear the tested frequencies used in experiments. Here, extracted ABRs showed typical ABR peaks indicating neuronal responses to sound at several levels of the auditory brainstem, and thereby intact hearing of tested stimuli. Since extracellular recordings can pick up ABR signals, ABR protocols can be useful to routinely add to single or multiunit recordings in the DCN especially when using older mice on a c57BL/6J background. Recently, new cre-lines expressing channelrhodopsin on a CBA background, with preserved hearing throughout adult life, have been developed (Lyngholm and Sakata, 2019). Lyngholm and Sakata (2019) show that CBA mice expressing ChR2 coupled to the parvalbumin promoter could excite cortical narrow spiking neurons (inhibitory interneurons) on light stimulation, and inhibit broad spiking units as effectively as in mice with a C57 background. On the other hand, a benefit of using the c57BL/6J mouse line is that it is more susceptible to acoustic overexposure than other strains (Willott and Erway, 1998; Davis et al., 2001) and thereby a suitable animal model for studying noise-induced tinnitus.

While several studies have applied general promoters to achieve optogenetic control of the DCN (Shimano et al., 2013; Darrow et al., 2015; Hight et al., 2015) there is still a lack of studies showing subpopulation control of the DCN in vivo. The CaMKIl $\alpha$ promoter is often used for targeting excitatory neurons in the neocortex and hippocampus (Wang et al., 2013), but here, we found that the CaMKII $\alpha$ promoter, for the viral constructs tested, would target morphologically different cell types within the DCN. This is in agreement with studies of the olfactory bulb, where CaMKII $\alpha$-GFP-positive neurons co-localize with GABA immunoreactivity (Wang et al., 2013). Still, to decrease activity of DCN excitatory neurons would be highly interesting for alleviating tinnitus. In slice preparations, vesicular glutamate transporter 2 (VGluT2) transgenic mice have already been used for targeting DCN fusiform and stellate cells to investigate control of DCN fusiform cell activity (Apostolides and Trussell, 2013a). Inhibitory DCN activity has been investigated using glycine transporter 2 (GlyT2-cre mice) for controlling DCN cartwheel cell firing in vitro (Apostolides and Trussell, 2013b; Lu and Trussell, 2016). Also the GABA/glycine transporter (VGAT) promoter has been used to excite inhibitory interneurons to study inhibitory neurotransmission in slices of the VCN (Xie and Manis, 2014), for example.

Attempting to silence DCN units responding to sound, using light stimulation of $\mathrm{CaMKIl} \alpha$-eArch3.0 expressing neurons showed that units could be inhibited using eArch3.0, but also that green light exposure generated a distinct delay in response-onset to sound. The delay was consistently around $20 \mathrm{~ms}$ suggesting polysynaptic activity, possibly from the recruitment of complex spiking cartwheel cells that can respond with 20 ms delay to pure tone stimulation (Parham et al., 2000). Also PSTH of unidentified neurons with a $40 \mathrm{~ms}$ delay between initial and basal firing have been reported for guinea pigs (Robertson and Mulders, 2018). Our experiment could not identify the type of unit responsible for this delay, but it shows that silencing CaMKII $\alpha$ -
eArch3.0 expressing neurons is not enough to disrupt sound generating activity in the DCN circuit. Some studies have pointed to technical problems when using the proton pump eArch3.0, as it may affect intracellular $\mathrm{pH}$ of presynaptic membranes and promote neurotransmitter release if light is applied continuously for several minutes (Mahn et al., 2016). Mahn et al. (2016) showed that $5 \mathrm{~min}$ of continuous eArch3.0 activity significantly increased the EPSC rate (Mahn et al., 2016). Thereby, eArch3.0 may not be the most appropriate tool for inhibiting neurons of the DCN for longer time periods. Here, we found that applying green light stimulation in blocks of $20 \mathrm{~s}$ was sufficient to alter temporal coding of some units and silence others. An advantage of green light stimulation, compared with blue, is that green wavelength light can penetrate deeper into tissue without being scattered. For example, it has been shown in a modeling study that green light penetrates skin tissue twice as deep as blue light (Ash et al., 2017). Thereby, the green light applied here should be adequate for illuminating the DCN, and not be the reason for failed neuronal silencing in units showing delayed sound response. Still, the placement of the recording electrode could influence our findings as we are only sampling local neurons according to the probe location. As we describe in methods, we adjusted our coordinates to the animal skull size and recorded at three different depths to cover an as large as possible region of the DCN for each animal, without inserting the probe at multiple ML/AP locations or aspirating the cerebellum, as done in other rodent studies (Kaltenbach and Zhang, 2007; Shore et al., 2008; Finlayson and Kaltenbach, 2009; Koehler et al., 2011; Dehmel et al., 2012; Manzoor et al., 2012). Furthermore, spontaneously firing neurons that decreased or increased firing on green light stimulation were recorded all along the probe, showing that they were not from any specific DCN layer. An interesting finding from CaMKIl $\alpha$-eArch3.0 experiments was that many neurons not responding directly to brief sound of $5-15 \mathrm{kHz}$ were indirectly affected by silencing CaMKII $\alpha$-eArch3.0-positive neurons in the DCN. Also, that inhibiting neurons expressing CaMKIl $\alpha$-eArch 3.0 can be used to modulate both high-frequency and low-frequency firing neurons not responding directly to sound, and that this modulation was bidirectional. This suggests that $\mathrm{CaMKII} \alpha$ expressing neurons can modulate excitation/inhibition ratios to some extent (see Fig. $5 C$ ). Here, stimulating the DCN with longer sound at additional frequencies would clarify what type of units respond by decreasing or increasing spike activity when inhibiting CaMKIl $\alpha+$ DCN neurons.

One of the disadvantages of optogenetic stimulation is that high intensity laser stimulation can increase the temperature of the surrounding tissue and that many neuronal responses are temperature-dependent (Owen et al., 2019). Especially $15 \mathrm{~mW} / \mathrm{mm}^{2}$ green light for $1-5 \mathrm{~s}$ can alter firing rate and generate heat-sensitive ion currents (Owen et al., 2019). Here, at the light intensity of 5-7 mW/ $\mathrm{mm}^{2}$, we did not observe altered firing rate to sound in the presence of blue light pulses or $20 \mathrm{~s}$ green light stimulation in control animals, indicating that the changes we observed in the injected animals were attributed to the expression and activation of opsins. 
A limitation of our study is that we did not assess the best frequency of units. Thereby the sound stimulus will not display full firing potential nor specific firing patterns (such as pauser, onset, build-up units). However, as light stimulation also was brief, it allows for more direct comparison between modulation of unit activity in responses to sound or after exciting DCN units with light. By not assessing the best frequency and not presenting sound of different frequencies at different intensities also prevents us from building frequency response areas (Palmer et al., 2013) for each unit. However, the linear disposition of the channels in the probe allowed recording of units along all the DCN layers, which is known to aggregate different types of cells (Oertel and Young, 2004). One of the interesting findings of this study was that sound pulses alone elicited more spikes than sound+light stimuli. There are different possible explanations for this, such as inhibitory neurons express opsins and thus the observed decrease could be part of motifs of feed-forward inhibition (Roberts and Trussell, 2010). Or, the fact that the light stimulus is slightly longer and starts earlier than the sound pulse, thereby the light pulse could immediately activate inhibitory neurons just before the sound pulse, which would decrease the probability of the circuitry to be activated compared with the sound pulse alone. Also, for excitatory neurons expressing ChR2, the unit could be activated by the light pulse, and next be in a non-excitable state when the sound pulse is presented. This "light-on-sound" masking was shown before (Hernandez et al., 2014), where expressing ChR2 in cochlear spiral ganglion neurons and presenting the sound stimulus embedded in blue light stimulation masked the evoked ABR, although not completely abolished (Hernandez et al., 2014).

Our work also shows for the first time that the Chrna2cre line targets two different cell populations of the VCN; both putative T-stellate cells and bushy cells of the VCN. T-stellate cells, also called Type I/multipolar/choppers, are excitatory neurons of the VCN with diverse connectivity but project mainly to the contralateral inferior colliculus (for review, see Oertel et al., 2011). T-stellate cells have been shown to have axon collaterals that go into the deep layers of DCN without reaching the fusiform cell layer (Oertel et al., 2011) which is similar to the diffuse axon terminals we observed (Fig. 7A; and CLARITY data). Our virus labeling also corroborates the indication that T-stellate cells provide input to the ipsilateral LSO, as we observed strong labeling of the ipsilateral LSO. However, bushy cells of the AVCN also project to the ipsilateral LSO, making additional experiments needed to confirm the degree of such connectivity. It is also known that Tstellate cells are modulated by nicotinergic agonists while D-stellate cells are not (Fujino and Oertel, 2001), in agreement with our cre-line that specifically targets neurons with nicotinic receptors containing the Chrna2. Recently, T-stellate cells were shown to innervate neighboring Tstellate cells responding to similar sound frequencies, regulating local excitation through nitric oxide-dependent plasticity mechanisms (Cao et al., 2019). The Chrna2-cre line could possibly aid in studies of hyperexcitability in Tstellate cells relating to hearing loss and/or tinnitus
(Coomber et al., 2014; Cao et al., 2019). Here, using Chrna2-cre mice and cre-dependent viral constructs we could excite DCN units that did not respond directly to sound, but responded temporally precisely to light stimulation of the VCN, suggesting that specific circuits may be targeted using these animals. We found no disruption of hearing on optogenetic stimulation, but specifically altered network activity compared with brief sound stimulation, including delayed responses and disinhibition of activity. Interestingly, we also found altered spiking activity for units not responding directly to sound. Furthermore, stimulation of bushy cells may especially be useful for studies of the calyx of Held presynaptic release and/or sound localization studies using the Chrna2-cre line. Together, these results open up for more detailed control of cochlear nucleus circuit output in vivo and novel tools for studying tinnitus mechanisms.

In conclusion, optogenetic stimulation of $\mathrm{CaMKII} \alpha+$ DCN neurons or Chrna2 expressing VCN neurons can be used to manipulate unit firing of the DCN and to modulate response to sound. We found that the CaMKII $\alpha$ promoter can be used to optogenetically lower or scramble timing of neuronal activity of the DCN but not to specifically activate the $\mathrm{DCN}$ in a temporally precise manner or increase response to sound. As the DCN is implicated in tinnitus, the CaMKIl $\alpha$ promoter could provide a useful, low-cost (compared with transgenic animals) option for lowering overall DCN activity. Furthermore, the Chrna2 promoter provides an interesting genetic tool as smaller and more specific network activity modulation may be achieved compared with sound stimulation.

\section{References}

Achor L, Starr A (1980) Auditory brain stem responses in the cat. i. intracranial and extracranial recordings. Electroencephalogr Clin Neurophysiol 48:154-173.

Apostolides PF, Trussell LO (2013a) Rapid, activity-independent turnover of vesicular transmitter content at a mixed glycine/GABA synapse. J Neurosci 33:4768-4781.

Apostolides PF, Trussell LO (2013b) Regulation of interneuron excitability by gap junction coupling with principal cells. Nat Neurosci 16:1764-1772.

Ash C, Dubec M, Donne K, Bashford T (2017) Effect of wavelength and beam width on penetration in light-tissue interaction using computational methods. Lasers Med Sci 32:1909-1918.

Baizer JS, Manohar S, Paolone NA, Weinstock N, Salvi RJ (2012) Understanding tinnitus: the dorsal cochlear nucleus, organization and plasticity. Brain Res 1485:40-53.

Boyden ES, Zhang F, Bamberg E, Nagel G, Deisseroth K (2005) Millisecond-timescale, genetically targeted optical control of neural activity. Nat Neurosci 8:1263-1268.

Cao XJ, Lin L, Sugden AU, Connors BW, Oertel D (2019) Nitric oxidemediated plasticity of interconnections between t-stellate cells of the ventral cochlear nucleus generate positive feedback and constitute a central gain control in the auditory system. J Neurosci 39:6095-6107.

Caswell TA, Droettboom M, Hunter J, Firing E, Lee A, Klymak J, Stansby D, Sales de Andrade E, Hedegaard Nielsen J, Varoquaux N, Root B, Hoffmann T, Elson P, May R, Dale D, Lee JJ, Seppänen JK, McDougall D, Straw A, Hobson P, et al. (2019) matplotlib/matplotlib v2.2.4. Available from https://zenodo.org/record/2669103\#. YBmaZOhKg2w. Zenodo. 
Chow BY, Han X, Dobry AS, Qian X, Chuong AS, Li M, Henninger MA, Belfort GM, Lin Y, Monahan PE, Boyden ES (2010) High-performance genetically targetable optical neural silencing by lightdriven proton pumps. Nature 463:98-102.

Coomber B, Berger JI, Kowalkowski VL, Shackleton TM, Palmer AR, Wallace MN (2014) Neural changes accompanying tinnitus following unilateral acoustic trauma in the guinea pig. Eur $\mathrm{J}$ Neurosci 40:2427-2441.

Coomber B, Kowalkowski VL, Berger JI, Palmer AR, Wallace MN (2015) Modulating central gain in tinnitus: changes in nitric oxide synthase in the ventral cochlear nucleus. Front Neurol 6:53.

Darrow KN, Slama MCC, Kozin ED, Owoc M, Hancock K, Kempfle J, Edge A, Lacour S, Boyden E, Polley D, Brown MC, Lee DJ (2015) Optogenetic stimulation of the cochlear nucleus using channelrhodopsin-2 evokes activity in the central auditory pathways. Brain Res 1599:44-56.

Davis RR, Newlander J, Ling X-B, Cortopassi GA, Krieg EF, Erway LC (2001) Genetic basis for susceptibility to noise-induced hearing loss in mice. Hear Res 155:82-90.

Dehmel S, Pradhan S, Koehler S, Bledsoe S, Shore S (2012) Noise overexposure alters long-term somatosensory-auditory processing in the dorsal cochlear nucleus-possible basis for tinnitus-related hyperactivity? J Neurosci 32:1660-1671.

Devor A (2000) Is the cerebellum like cerebellar-like structures? Brain Res Brain Res Rev 34:149-156.

Finlayson PG, Kaltenbach JA (2009) Alterations in the spontaneous discharge patterns of single units in the dorsal cochlear nucleus following intense sound exposure. Hear Res 256:104-117.

Franklin K, Paxinos G (2008) The mouse brain in stereotaxic coordinates. San Diego: Academic Press.

Fujino K, Oertel D (2001) Cholinergic modulation of stellate cells in the mammalian ventral cochlear nucleus. J Neurosci 21:73727383.

Grossan M, Peterson DC (2017) Tinnitus. Treasure Island: StatPearls Publishing.

Han BI, Lee HW, Kim TY, Lim JS, Shin KS (2009) Tinnitus: characteristics, causes, mechanisms, and treatments. J Clin Neurol 5:1119.

Henry KR (1979) Auditory brainstem volume-conducted responses: origins in the laboratory mouse. J Am Aud Soc 4:173-178.

Hernandez VH, Gehrt A, Reuter K, Jing Z, Jeschke M, Mendoza Schulz A, Hoch G, Bartels M, Vogt G, Garnham CW, Yawo H, Fukazawa Y, Augustine GJ, Bamberg E, Kügler S, Salditt T, de Hoz L, Strenzke N, Moser T (2014) Optogenetic stimulation of the auditory pathway. J Clin Invest 124:1114-1129.

Hight AE, Kozin ED, Darrow K, Lehmann A, Boyden E, Brown MC, Lee DJ (2015) Superior temporal resolution of chronos versus channelrhodopsin-2 in an optogenetic model of the auditory brainstem implant. Hear Res 322:235-241.

Hilscher MM, Leão RN, Edwards SJ, Leão KE, Kullander K (2017) Chrna2-martinotti cells synchronize layer 5 type a pyramidal cells via rebound excitation. PLOS Biol 15:e2001392.

Hunter JD (2007) Matplotlib: a 2D graphics environment. Comput Sci Eng 9:90-95.

Jewett DL (1970) Volume-conducted potentials in response to auditory stimuli as detected by averaging in the cat. Electroencephalogr Clin Neurophysiol 28:609-618.

Jones E, Oliphant T, Peterson P (2001) SciPy: open source scientific tools for Python. Available from http://www.scipy.org

Kaltenbach JA, Zhang J (2007) Intense sound-induced plasticity in the dorsal cochlear nucleus of rats: evidence for cholinergic receptor upregulation. Hear Res 226:232-243.

Kaltenbach JA, Zhang J, Finlayson P (2005) Tinnitus as a plastic phenomenon and its possible neural underpinnings in the dorsal cochlear nucleus. Hear Res 206:200-226.

Kawaguchi Y, Kondo S (2002) Parvalbumin, somatostatin and cholecystokinin as chemical markers for specific gabaergic interneuron types in the rat frontal cortex. J Neurocytol 31:277-287.
Koehler SD, Pradhan S, Manis PB, Shore SE (2011) Somatosensory inputs modify auditory spike timing in dorsal cochlear nucleus principal cells. Eur J Neurosci 33:409-420.

Kraus K, Ding D, Jiang H, Lobarinas E, Sun W, Salvi R (2011) Relationship between noise-induced hearing-loss, persistent tinnitus and growth-associated protein-43 expression in the rat cochlear nucleus: does synaptic plasticity in ventral cochlear nucleus suppress tinnitus? Neuroscience 194:309-325.

Leão RN, Mikulovic S, Leão KE, Munguba $H$, Gezelius $H$, Enjin A, Patra K, Eriksson A, Loew LM, Tort ABL, Kullander K (2012) OLM interneurons differentially modulate $\mathrm{CA} 3$ and entorhinal inputs to hippocampal CA1 neurons. Nat Neurosci 15:1524-1530.

Lein ES, Hawrylycz MJ, Ao N, Ayres M, Bensinger A, Bernard A, Boe AF, Boguski MS, Brockway KS, Byrnes EJ, Chen L, Chen L, Chen TM, Chin MC, Chong J, Crook BE, Czaplinska A, Dang CN, Datta $S$, Dee NR, et al. (2007) Genome-wide atlas of gene expression in the adult mouse brain. Nature 445:168-176.

Lev A, Sohmer H (1972) Sources of averaged neural responses recorded in animal and human subjects during cochlear audiometry (electro-cochleogram). Arch Klin Exp Ohren Nasen Kehlkopfheilkd 201:79-90.

Levine RA (1999) Somatic (craniocervical) tinnitus and the dorsal cochlear nucleus hypothesis. Am J Otolaryngol 20:351-362.

Lu HW, Trussell LO (2016) Spontaneous activity defines effective convergence ratios in an inhibitory circuit. J Neurosci 36:32683280.

Lyngholm D, Sakata S (2019) Cre-dependent optogenetic transgenic mice without early age-related hearing loss. Front Aging Neurosci 11:29.

Mahn M, Prigge M, Ron S, Levy R, Yizhar O (2016) Biophysical constraints of optogenetic inhibition at presynaptic terminals. Nat Neurosci 19:554-556.

Manzoor NF, Licari FG, Klapchar M, Elkin RL, Gao Y, Chen G, Kaltenbach JA (2012) Noise-induced hyperactivity in the inferior colliculus: its relationship with hyperactivity in the dorsal cochlear nucleus. J Neurophysiol 108:976-988.

Mikulovic S, Restrepo CE, Hilscher MM, Kullander K, LeÃ£o RN (2015) Novel markers for OLM interneurons in the hippocampus. Front Cell Neurosci 9:201.

Mikulovic S, Restrepo CE, Siwani S, Bauer P, Pupe S, Tort ABL, Kullander K, Leão RN (2018) Ventral hippocampal OLM cells control type 2 theta oscillations and response to predator odor. Nat Commun 9:3638.

Milinkeviciute G, Muniak MA, Ryugo DK (2017) Descending projections from the inferior colliculus to the dorsal cochlear nucleus are excitatory. J Comp Neurol 525:773-793.

Noben-Trauth K, Zheng QY, Johnson KR (2003) Association of cadherin 23 with polygenic inheritance and genetic modification of sensorineural hearing loss. Nat Genet 35:21-23.

Ochiishi T, Yamauchi T, Terashima T (1998) Regional differences between the immunohistochemical distribution of ca2+/calmodulindependent protein kinase ii $\alpha$ and $\beta$ isoforms in the brainstem of the rat. Brain Res 790:129-140.

Oertel D, Young ED (2004) What's a cerebellar circuit doing in the auditory system? Trends Neurosci 27:104-110.

Oertel D, Wright S, Cao X-J, Ferragamo M, Bal R (2011) The multiple functions of $t$ stellate/multipolar/chopper cells in the ventral cochlear nucleus. Hear Res 276:61-69.

Owen SF, Liu MH, Kreitzer AC (2019) Thermal constraints on in vivo optogenetic manipulations. Nat Neurosci 22:1061-1065.

Palmer AR, Shackleton TM, Sumner CJ, Zobay O, Rees A (2013) Classification of frequency response areas in the inferior colliculus reveals continua not discrete classes. J Physiol 591:4003-4025.

Parham K, Bonaiuto G, Carlson S, Turner JG, D'Angelo WR, Bross LS, Fox A, Willott JF, Kim DO (2000) Purkinje cell degeneration and control mice: responses of single units in the dorsal cochlear nucleus and the acoustic startle response. Hear Res 148:137-152.

Parras GG, Nieto-Diego J, Carbajal GV, Valdés-Baizabal C, Escera C, Malmierca MS (2017) Neurons along the auditory pathway 
exhibit a hierarchical organization of prediction error. Nat Commun 8:2148.

Perry S, Gezelius H, Larhammar M, Hilscher MM, Lamotte d'Incamps B, Leao KE, Kullander K (2015) Firing properties of renshaw cells defined by chrna2 are modulated by hyperpolarizing and small conductance ion currents ih and isk. Eur $\mathrm{J}$ Neurosci 41:889-900.

Roberts MT, Trussell LO (2010) Molecular layer inhibitory interneurons provide feedforward and lateral inhibition in the dorsal cochlear nucleus. J Neurophysiol 104:2462-2473.

Robertson D, Mulders WH (2018) Cholinergic responses of acoustically-characterized cochlear nucleus neurons: an in vivo iontophoretic study in guinea pig. Hear Res 367:97-105.

Rossant C, Kadir SN, Goodman DFM, Schulman J, Hunter MLD, Saleem AB, Grosmark A, Belluscio M, Denfield GH, Ecker AS, Tolias AS, Solomon S, Buzsaki G, Carandini M, Harris KD (2016) Spike sorting for large, dense electrode arrays. Nat Neurosci 19:634-641.

Schneider CA, Rasband WS, Eliceiri KW (2012) NIH image to ImageJ: 25 years of image analysis. Nat Methods 9:671-675.

Shimano T, Fyk-Kolodziej B, Mirza N, Asako M, Tomoda K, Bledsoe S, Pan ZH, Molitor S, Holt AG (2013) Assessment of the AAV-mediated expression of channelrhodopsin-2 and halorhodopsin in brainstem neurons mediating auditory signaling. Brain Res 1511:138-152.

Shore SE, Koehler S, Oldakowski M, Hughes LF, Syed S (2008) Dorsal cochlear nucleus responses to somatosensory stimulation are enhanced after noise-induced hearing loss. Eur $\mathrm{J}$ Neurosci $27: 155-168$.
Shore SE, Roberts LE, Langguth B (2016) Maladaptive plasticity in tinnitus - triggers, mechanisms and treatment. Nat Rev Neurol 12:150-160.

Siegle JH, López AC, Patel YA, Abramov K, Ohayon S, Voigts J (2017) Open-Ephys: an open-source, plugin-based platform for multichannel electrophysiology. J Neural Eng 14:e045003.

Siwani S, França ASC, Mikulovic S, Reis A, Hilscher MM, Edwards SJ, Leão RN, Tort ABL, Kullander K (2018) OLM $\alpha 2$ cells bidirectionally modulate learning. Neuron 99:404-412.e3.

Tzounopoulos T (2008) Mechanisms of synaptic plasticity in the dorsal cochlear nucleus: plasticity-induced changes that could underlie tinnitus. Am J Audiol 17:S170-S175.

Van Der Walt S, Colbert SC, Varoquaux G (2011) The NumPy array: a structure for efficient numerical computation. Comput Sci Eng 13:22-30.

Wang X, Zhang C, Szábo G, Sun Q-Q (2013) Distribution of CaMKIl $\alpha$ expression in the brain in vivo, studied by CaMKII $\alpha$-GFP mice. Brain Res 1518:9-25.

Willott JF, Erway LC (1998) Genetics of age-related hearing loss in mice. IV. Cochlear pathology and hearing loss in 25 BXD recombinant inbred mouse strains. Hear Res 119:27-36.

Winer JA, Prieto JJ (2001) Layer v in cat primary auditory cortex (ai): cellular architecture and identification of projection neurons. J Comp Neurol 434:379-412.

Xie R, Manis PB (2014) GABAergic and glycinergic inhibitory synaptic transmission in the ventral cochlear nucleus studied in VGAT channelrhodopsin-2 mice. Front Neural Circuits 8:84. 